\title{
Synthesis, Characterization, Single Crystal Structural Studies, Antibacterial Activity and DFT Investigations of 2-Chloro-5-Ethoxy-3,6- Bis(Methylamino)-1,4-Benzoquinone
}

\author{
Gautam BPS ${ }^{1}$, Dani RK' ${ }^{1}$, Prasad RL¹, Srivastava $\mathbf{M}^{2}$, Yadav RA ${ }^{2^{*}}$ and Gondwal $\mathbf{M}^{3}$
}

${ }^{1}$ Department of Chemistry, Banaras Hindu University, Varanasi, UP, India

${ }^{2}$ Department of Physics, Banaras Hindu University, Varanasi, UP, India

${ }^{3}$ Department of Chemistry, Hemwati Nandan Bahuguna Garhwal University, Srinagar (Garhwal), Uttrakhand, India

\begin{abstract}
A new compound 2-chloro-5-ethoxy-3,6-bis(methylamino)-1,4-benzoquinone (cemb) has been synthesized and characterized by various physicochemical techniques. The compound crystallizes in the triclinic system with the space group P-1 and contains extended hydrogen bonding interaction providing supramolecular architecture. The compound contains $\mathrm{CH}^{\cdots} \pi$ and oxygen $\cdots \pi$ interaction which stabilizes the crystal structure and is also responsible for the semiconducting behavior. The DFT calculations in the gaseous phase of cemb with B3LYP hybrid functional show good correlation with experimental results (single crystal XRD data) and are used to predict the molecular properties. Excluding the side ethyl group, the structure possesses $C_{s}$ symmetry. The ring breathing mode of vibration is found to have frequency $\left(551 \mathrm{~cm}^{-1}\right)$ which is much lower as compared to the corresponding mode of benzene $\left(991 \mathrm{~cm}^{-1}\right)$. The bioefficacy of the compound has been examined against the growth of bacteria in vitro to evaluate antimicrobial potential. The pphotoluminescence properties indicate that the compound has characteristic fluorescence emissions. The HOMO and LUMO energy gap reveals that the energy gap reflects the chemical activity of the molecule.
\end{abstract}

Keywords: 1,4-Benzoquinone derivative; Crystal structure; Solid state electrical conductivity; Antibacterial activity; DFT calculations

\section{Introduction}

Naturally occurring compounds containing a quinone group are involved in various bioenergetic processes as electron-transport agents. Some quinones play important roles in the biochemistry of energy production and serve as vital links during electron transport. These compounds have attracted considerable attention because of their biological activity and chemotherapeutic values [1]. Various quinone containing oxygenated aromatic rings show biological activities [2]. Quinones are found in all respiring animal and plant cells where they function primarily as components of the electron transport chains involved in cellular respiration [3] and photosynthesis [4]. These compounds occur in other natural contexts and as pollutants [4-8]. Quinones form an important class of toxic metabolites and paradoxically can be either mutagenic and, therefore, are potentially carcinogenic, or effective anticancer agents [9]. Some quinones possibly play roles in the cellular defence since they effectively inhibit growth of bacteria, fungi and/or parasites [10-13].

Quinones are a large class of compounds endowed with rich and fascinating chemistry [14]. They are widely distributed in the natural world [15], being found in bacteria, plants and arthropods and hence, quinones are ubiquitous to living systems. Their role as electron transfer agents in primary metabolic processes like photosynthesis and respiration is vital to human life. A large number of chemical derivatives with 1,4-benzoquinone as the basic subunit exhibit prominent pharmacological applications such as antibiotic, [16,17] antitumor, [18-21] antimalarial, [22] antineoplastic, [23] anticoagulant [24] and herbicidal activities [25]. Coordination chemistry of quinones is also quite rich from the perspective of designing magnetic materials [26] and understanding photo physical properties [27]. The chemistry of quinones and hydroquinones is of interest because of their involvement in the electron and hydrogen transfer reactions in various processes including the biological energy conversion reactions such as respiration and photosynthesis [28-30]. For the antibacterial agents often used, quinone compounds are tetracyclines, new quinolones, anthracyclines, and mitomycins. More than 2000 naturally occurring quinones, likeanthraquinones, naphthoquinones, and benzoquinones are now known and are widely distributed in nature as pigments and intermediates in the cellular respiration and photosynthesis [31,32].

In view of the above, it is of great interest to investigate the derivatives of benzoquinone and to study the mode of bonding and structure. The present paper reports the synthesis, spectral, structural and DFT studies of 2-chloro-5-ethoxy-3,6-bis(methylamino)-1,4benzoquinone (cemb).

\section{Experimental Details}

\section{Chemicals and starting materials}

Commercial reagents were used without further purification and all experiments were carried out in open atmosphere. The solvents were dried and distilled before use, following the standard procedures.

\section{Physical measurements}

*Corresponding author: Yadav RA, Department of Physics, Banaras Hindu University, Varanasi-221005, UP, India, Tel: +91542-2368593; E-mail: rayadav@ bhu.ac.in

Received July 07, 2015; Accepted August 21, 2015; Published August 24, 2015

Citation: Gautam BPS, Dani RK, Prasad RL, Srivastava M, Yadav RA, et al. (2015) Synthesis, Characterization, Single Crystal Structural Studies, Antibacterial Activity and DFT Investigations of 2-Chloro-5-Ethoxy-3,6-Bis(Methylamino)-1,4-Benzoquinone. Pharm Anal Acta 6: 418. doi:10.4172/21532435.1000418

Copyright: (C) 2015 Gautam BPS, et al. This is an open-access article distributed under the terms of the Creative Commons Attribution License, which permits unrestricted use, distribution, and reproduction in any medium, provided the original author and source are credited. 
Citation: Gautam BPS, Dani RK, Prasad RL, Srivastava M, Yadav RA, et al. (2015) Synthesis, Characterization, Single Crystal Structural Studies, Antibacterial Activity and DFT Investigations of 2-Chloro-5-Ethoxy-3,6-Bis(Methylamino)-1,4-Benzoquinone. Pharm Anal Acta 6: 418. doi:10.4172/21532435.1000418

Page 2 of 10

Carbon, hydrogen, nitrogen contents were estimated on a CHN Model CE-440 Analyser and on an Elementar Vario EL III Carlo Erba 1108. IR spectra were recorded in the $4000-400 \mathrm{~cm}^{-1}$ region in $\mathrm{KBr}$ pellets on a Perkin Elmer/Varian Excalibur 3100 FT-IR spectrophotometer. ${ }^{1} \mathrm{H}$ and ${ }^{13} \mathrm{C}$ NMR spectra were recorded in DMSO- $d_{6}$ on a JEOL AL300 FT-NMR spectrophotometer. The electrochemical experiments were performed with a CHI-660C (CH Instruments, USA), using threeelectrode system, platinum wire as counter electrode (area $\sim 8.0 \mathrm{~cm}^{2}$ ) and $\mathrm{Hg} / \mathrm{HgO}$ was used as the reference electrode. Electrical conductance of pressed pellets was measured by a conventional two-probe method in the $313-443 \mathrm{~K}$ range with contacts made on the pellet surfaces by means of silver paint. A Keithley $236 \mathrm{SMU}$ was used to measure the sample resistance. The fluorescent data were collected at room temperature with a Varian CARYECLIPSE spectrophotometer. Six bacterial pathogens Salmonella typhi, Shigella flexneri, Staphylococcus aureus, Aeromonas hydrophila, E. coli and Enterococcus faecalis were used to test the antibacterial activity of the compound. Commercial antibacterial drugs, streptomycin sulphate and chloramphenicol (Himedia) in same concentration of 10-100 $\mu \mathrm{g} /$ disc are used to compare effectiveness of the test compounds.

\section{Synthesis of cemb}

For the synthesis of cemb, sodium acetate $(1.148 \mathrm{~g}, 14 \mathrm{mmol})$ was added pinch by pinch within $20 \mathrm{~min}$ to a reaction mixture of chloranil (0.492 g, $2 \mathrm{mmol})$, which was added drop wise to methylamine hydrochloride $(0.270 \mathrm{~g}, 4 \mathrm{mmol})$ in $15 \mathrm{ml}$ of absolute ethanol with constant stirring for $1 \mathrm{~h}$ and refluxed $4 \mathrm{~h}$ at $65^{\circ} \mathrm{C}$. The solid separated was filtered off, washed out successively with ethanol-water mixture (50:50 $\mathrm{v} / \mathrm{v})$ and air dried. The precipitates were suspended in ethanol $(20 \mathrm{~mL})$ to which pyridine $(1 \mathrm{~mL})$ was added and refluxed for $1 \mathrm{~h}$ at $65^{\circ} \mathrm{C}$. The resulting clear solution was filtered and kept for crystallization. Single crystals of cemb suitable for X-ray analysis were obtained by slow evaporation of the solutions over a period of 25 days (Scheme 1). Grey color crystals were obtained with: Yield: $72 \%$; m.p $>290^{\circ} \mathrm{C}$; Anal. Found: C, 49.72; $\mathrm{H}, 5.46 ; \mathrm{N}, 11.05 \%$. Calc. for $\mathrm{C}_{10} \mathrm{H}_{13} \mathrm{ClN}_{2} \mathrm{O}_{3}$ (244.67): C, 49.09; $\mathrm{H}, 5.36 ; \mathrm{N}, 11.45 \%$. The crystal structure was confirmed by XRD.

\section{Antibacterial assay}

The antibacterial assay was done according to the Bayer method [33]. The test compounds were dissolved in DMSO to a final concentration of $5 \mathrm{mg} / \mathrm{mL}$. Sterilized Whatman no. 1 filter paper discs (5 $\mathrm{mm}$ ) were impregnated with different volume $(2,4,8,12,16$ and $20 \mu \mathrm{L}$ ) of compounds to get a final concentration of $10,20,40,60,80$ and $100 \mu \mathrm{g}$ per disc. Sterilized paper disc loaded with the $10 \mu \mathrm{L}$ of DMSO was taken as a control. The bacterial test pathogens were spread on fresh Müller Hinton Agar (MHA) plates with the help of cotton swabs to form an even lawn of the test bacteria. The filter paper disc impregnated with the test compounds were placed on the surface of the MHA plates seeded with test bacteria and the plates were incubated in a BOD Incubator (Caltan-152, Narang Scientific Works, New Delhi, India) for $24 \mathrm{~h}$ at $37 \pm 2^{\circ} \mathrm{C}$. The inhibition zones around each disc were

$$
\begin{aligned}
& \text { 每 }
\end{aligned}
$$

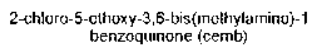

Scheme1: Synthesis of 2-chloro-5-ethoxy-3,6-bis(methylamino)-1,4benzoquinone (cemb). measured after $24 \mathrm{~h}$ of incubation.

\section{Crystal structure determination}

Crystals suitable for X-ray analysis of cemb were grown at the room temperature. The crystal structure data were collected on an Oxford Gemini diffractometer equipped with a CrysAlis CCD software using a graphite mono-chromated Mo $\mathrm{Ka}(\lambda=0.71073 \AA)$ radiation source at $293 \mathrm{~K}$ for cemb. Multiscan absorption correction was applied to the X-ray data collection and the structure was solved by direct methods (SHELXS-08) and refined against all data by full matrix leastsquare on $\mathrm{F}^{2}$ using anisotropic displacement parameters for all nonhydrogen atoms. All hydrogen atoms were included in the refinement at geometrically ideal position and refined with a riding model [34]. The MERCURY package and ORTEP-3 for Windows program were used for generating structures $[35,36]$. The details of data collection, structure solution and refinement are listed in Table 1. Selected bond lengths and angles are given in Table 2. ORTEP diagram of cemb with atom numbering schemes is shown in Figure 1A.The single crystal $\mathrm{X}$-ray diffraction studies indicate that the starting compound chloranil is converted to cemb during the course of reaction. The grown single crystals of cemb were analyzed by single crystal X-ray data. The bond lengths and angles in the ring are normal $[37,38]$. The structure is stabilized by intermolecular $\mathrm{N}-\mathrm{H} \cdots \mathrm{O}$ and $\mathrm{C}-\mathrm{H} \cdots \mathrm{O}$ hydrogen bonding occurring between hydrogen of substituted $\mathrm{N}$-methyl amine group to oxygen of nearby molecule and $\mathrm{CH} \cdots \pi$ interaction occurring between hydrogen of substituted ethoxy group to centroid of ring of a nearby molecule at a distance of $3.561 \AA$ and Oxygen $\cdots \pi$ at a distance of 3.557 Á producing linear chain structure. The structure is also stabilized by

\begin{tabular}{|c|c|}
\hline Empirical formula & $\mathrm{C}_{10} \mathrm{H}_{13} \mathrm{CIN}_{2} \mathrm{O}_{3}$ \\
\hline Formula weight & 244.67 \\
\hline $\mathrm{T}(\mathrm{K})$ & $293(2)$ \\
\hline$\Lambda(\mathrm{Mo} \mathrm{Ka})(\AA)$ & 0.71073 \\
\hline Crystal system & Triclinic \\
\hline Space group & $\mathrm{P}-1$ \\
\hline a $(\AA)$ & $7.5866(15)$ \\
\hline $\mathrm{b}(\AA)$ & $8.6898(18)$ \\
\hline$c(\AA)$ & $9.740(2)$ \\
\hline$\alpha\left({ }^{\circ}\right)$ & $91.172(18)$ \\
\hline$\beta\left({ }^{\circ}\right)$ & $112.09(2)$ \\
\hline$Y\left({ }^{\circ}\right)$ & $111.71(2)$ \\
\hline $\mathrm{V}\left(\AA^{3}\right)$ & $543.2(2)$ \\
\hline Z & 2 \\
\hline $\mathrm{D}_{\text {calc }}\left(\mathrm{mg} \mathrm{m}^{-3}\right)$ & 1.496 \\
\hline$\mu\left(\mathrm{mm}^{-1}\right)$ & 0.345 \\
\hline$F(000)$ & 256 \\
\hline Crystal size $\left(\mathrm{mm}^{3}\right)$ & $0.24 \times 0.21 \times 0.18$ \\
\hline$\theta$ range $\left({ }^{\circ}\right)$ & $3.11-29.00$ \\
\hline Index ranges & $\begin{array}{l}-10 \leq h \leq 9 \\
-11 \leq k \leq 11 \\
-12 \leq l \leq 13\end{array}$ \\
\hline Reflections collected & 3579 \\
\hline Independent reflections & 1205 \\
\hline Data/restraints/ parameters & $2894 / 0 / 145$ \\
\hline Goodness-of-fit on $F^{2}$ & 0.973 \\
\hline Final $R$ indices $w R_{2}[\mathrm{I}>2 \sigma(\mathrm{I})]\left(\mathrm{R}_{\text {int }}\right)$ & $0.0760,0.1516$ \\
\hline Final $R$ indices (all data) & $0.1561,0.1950$ \\
\hline Largest diff. peak/hole $\left(\mathrm{e} \AA^{-3}\right)$ & $0.357,-0.415$ \\
\hline
\end{tabular}

${ }^{a} R_{1}=\Sigma|| F_{0}|-| F_{\mathrm{c}}|| \Sigma\left|F_{\mathrm{o}}\right|,{ }^{\mathrm{b}} R_{2}=\left[\Sigma w\left(\left|F^{2}\right|-\left|F^{2}\right|\right)^{2} / \Sigma w \mid F^{2}{ }_{0}^{2}\right]^{1 / 2}$.

Table 1: Crystallographic data for cemb molecule. 
Citation: Gautam BPS, Dani RK, Prasad RL, Srivastava M, Yadav RA, et al. (2015) Synthesis, Characterization, Single Crystal Structural Studies, Antibacterial Activity and DFT Investigations of 2-Chloro-5-Ethoxy-3,6-Bis(Methylamino)-1,4-Benzoquinone. Pharm Anal Acta 6: 418. doi:10.4172/21532435.1000418

Page 3 of 10

\begin{tabular}{|c|c|c|c|c|c|}
\hline \multicolumn{3}{|c|}{ Bond lengths $(\AA)$} & \multicolumn{3}{|c|}{ Bond angles $\left(^{\circ}\right)$} \\
\hline & Exp. & Cal. & & Exp. & Cal. \\
\hline Cl-C11 & $1.737(4)$ & 1.74 & C9-O3-C4 & $117.3(4)$ & 118.2 \\
\hline O3-C9 & $1.325(6)$ & 1.32 & C3-N2-C8 & $129.3(4)$ & 129.5 \\
\hline O3-C4 & $1.414(5)$ & 1.42 & N2-C3-C4 & $128.4(4)$ & 128.8 \\
\hline O1-C5 & $1.246(4)$ & 1.25 & N2-C3-C2 & $112.9(3)$ & 112.3 \\
\hline N2-C3 & $1.326(5)$ & 1.34 & O2-C2-C1 & $123.5(4)$ & 123.9 \\
\hline N2-C8 & $1.436(5)$ & 1.44 & O2-C2-C3 & $116.5(4)$ & 116.8 \\
\hline O2-C2 & $1.249(4)$ & 1.26 & N1-C6-C5 & $111.5(3)$ & 112.2 \\
\hline N1-C6 & $1.326(5)$ & 1.34 & N1-C6-C1 & $129.2(4)$ & 129.4 \\
\hline N1-C7 & $1.445(5)$ & 1.45 & C6-N1-C7 & $130.9(4)$ & 130.5 \\
\hline C5-C6 & $1.523(6)$ & 1.52 & O1-C5-C4 & $123.2(4)$ & 123.6 \\
\hline C6-C1 & $1.376(5)$ & 1.36 & O1-C5-C6 & $117.1(3)$ & 117.6 \\
\hline
\end{tabular}

Table 2: Selected bond lengths $(\AA)$ and bond angles $\left(^{\circ}\right)$.

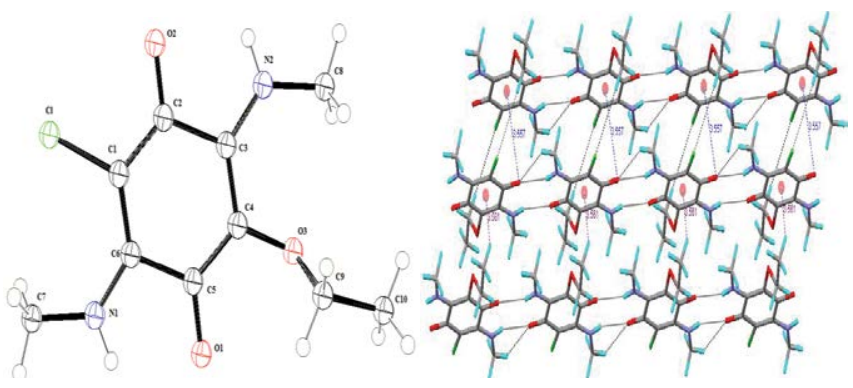

Figure 1: (A) ORTEP diagram of cemb molecule at $30 \%$ probability level. (B) $\mathrm{NH} \cdots \mathrm{O}, \mathrm{CH} \cdots \mathrm{O}, \mathrm{CH} \cdots \mathrm{Cl}, \mathrm{CH} \cdots \pi$ and Oxygen $\cdots \pi$ interactions leading to linear chain structure.

\begin{tabular}{|l|l|l|l|l|}
\hline $\mathrm{D}-\mathrm{H} \cdots \mathrm{A}$ & $\mathrm{d}(\mathrm{D}-\mathrm{H})$ & $\mathrm{d}(\mathrm{H} \cdots \mathrm{A})$ & $d(\mathrm{D} \cdots \mathrm{A})$ & $<(\mathrm{DHA})$ \\
\hline $\mathrm{N} 2-\mathrm{H} 2 \cdots \mathrm{O} 1$ & 0.859 & 2.155 & 2.917 & 147.59 \\
\hline $\mathrm{N} 1-\mathrm{H} 1 \cdots \mathrm{O} 2$ & 0.860 & 2.162 & 2.907 & 144.69 \\
\hline $\mathrm{C} 7-\mathrm{H} 01 \mathrm{D} \cdots \mathrm{O} 2$ & 0.961 & 2.642 & 3.228 & 119.69 \\
\hline $\mathrm{C} 9-\mathrm{H} 01 \mathrm{H} \cdots \mathrm{Cl} 1$ & 0.970 & 2.992 & 3.826 & 155.55 \\
\hline
\end{tabular}

Table 3: Intermolecular hydrogen bond parameters $\left[\AA\right.$ and $\left.{ }^{\circ}\right]$.

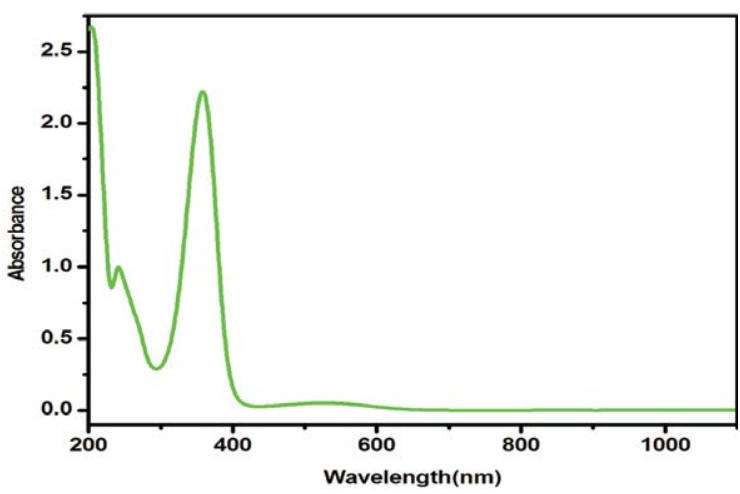

Figure 2: UV-Visible spectra.

intramolecular hydrogen bonding, i.e., $\mathrm{N}-\mathrm{H} \cdots \mathrm{O}, \mathrm{C}-\mathrm{H} \cdots \mathrm{O}$ and $\mathrm{C}-\mathrm{H} \cdots \mathrm{Cl}$ (Figure 1b). The geometrical parameters relevant for these interactions are given in Table 3. The above observed intermolecular interactions have stabilized the molecular crystal packing which leads to the formation of supramolecular architecture [39].

\section{UV-Vis spectra}

The experimental UV-vis spectrum of cemb in DMSO is shown in Figure 2. It shows a transition at $336.50 \mathrm{~nm}$.

\section{Photoluminescence emission spectra}

The photoluminescence emission spectrum of cemb was recorded in DMSO at room temperature (Figure 3). On excitation with a 335 $\mathrm{nm}$ laser source at room temperature, a strong fluorescence emission peaked at $426 \mathrm{~nm}$ is observed. The photoluminescence property shows that the compound has characteristic fluorescence emissions. The main chromospheres in the compound are the carbonyl and halogen groups and its conjugation degree is further enhanced by the amine group. The solution state photoluminescence property of compound in DMSO indicates that increase in the dilution of solution resulted in a blue shift and decrease in intensity at various concentrations due to weakening of the $\mathrm{CH} \cdots \pi$ and $\mathrm{CH} \cdots \mathrm{Cl}$ interactions, but the luminescence property persists even in dilute solution due to existence of the intermolecular interactions [40].

\section{Computational Details}

All calculations were performed using the Gaussian 03 and Gauss View 4.1 software [41]. The structure optimization of cemb was done using DFT method with the functional B3LYP and basis set 6-311 ++ G(d,p) [42]. The input geometry of the molecule for the DFT calculations was generated from the single crystal X-ray investigation carried out in the present study. The HOMO-LUMO energies, MEP, UV-vis spectra, linear polarizability and first hyperpolarizability were also calculated by the DFT method $[43,44]$. To facilitate the vibrational analysis potential energy distributions (PEDs) were also computed using the GAR2PED software.

\section{Results and Discussion}

Chloranil react with methylamine hydrochloride in presence of sodium acetate and pyridine to yield compound cemb. There is substitution of two methylamine and one ethoxy group with replacement of three chlorine atoms through a condensation reaction, which is confirmed by X-ray diffraction studies. The Scheme 1 depicts the formation of cemb. The compound is air stable, non-hygroscopic crystalline solids, which is insoluble in common organic solvents but soluble in DMF, DMSO and can be kept in desiccators over a prolonged period without any sign of decomposition with melting point at $>290^{\circ} \mathrm{C}$.

\section{Geometry optimization}

The optimized geometry (optimized energy -2037.988 a.u) of cemb are shown in Figure 4(A). There is good agreement between the geometrical parameters obtained by X-ray crystallography to those generated by DFT method as shown in Table 2. The slight disagreement in the bond lengths and angles are due to the fact that the experimental results are for the solid phase and the theoretical calculations are for the gas phase [45]. In the solid state, the existence of a crystal field along with the intermolecular interactions connect the molecules together, which results in the differences in geometrical parameters between the calculated and experimental values.

\section{UV-Vis spectra}

The DFT calculations were performed for the gas phase and DMSO solvent. Calculations predict an intense electronic transition at 328.24 $\mathrm{nm}$. 
Citation: Gautam BPS, Dani RK, Prasad RL, Srivastava M, Yadav RA, et al. (2015) Synthesis, Characterization, Single Crystal Structural Studies, Antibacterial Activity and DFT Investigations of 2-Chloro-5-Ethoxy-3,6-Bis(Methylamino)-1,4-Benzoquinone. Pharm Anal Acta 6: 418. doi:10.4172/21532435.1000418

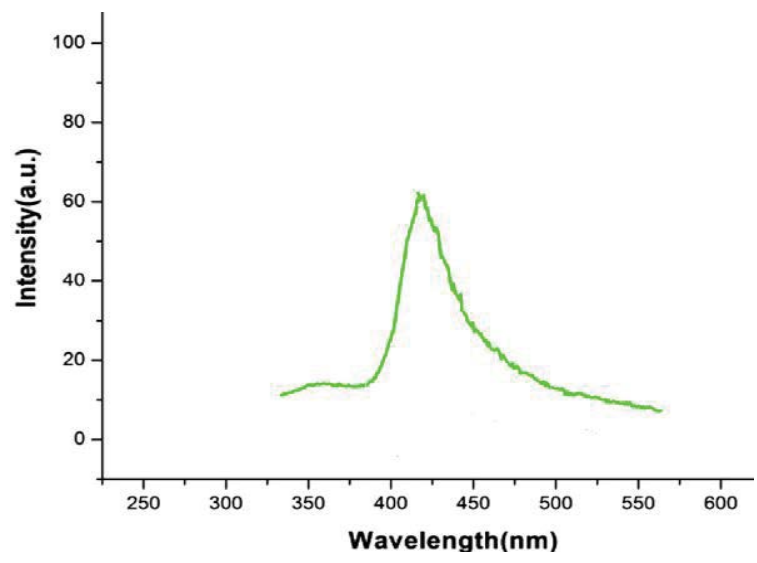

Figure 3: Fluorescence spectra.

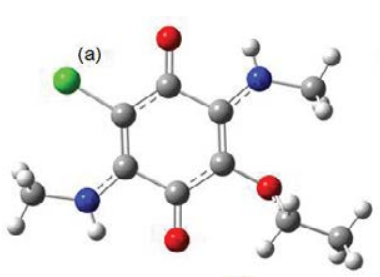

(c)

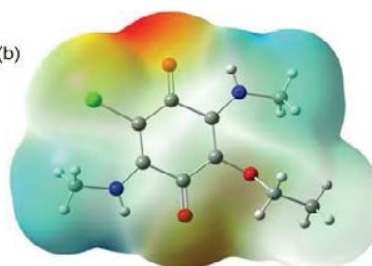

(d)
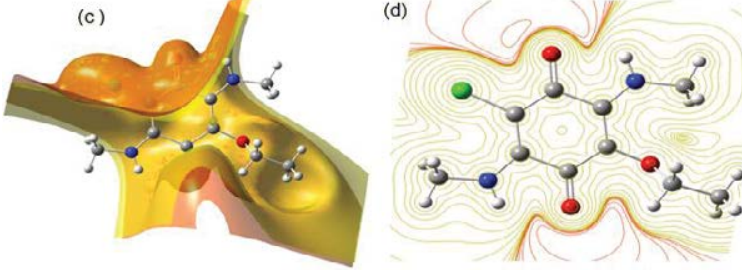

Figure 4: (A) Optimized structure (B) MEP plot (C) ESP plot and (D) Contour map of cemb molecule.

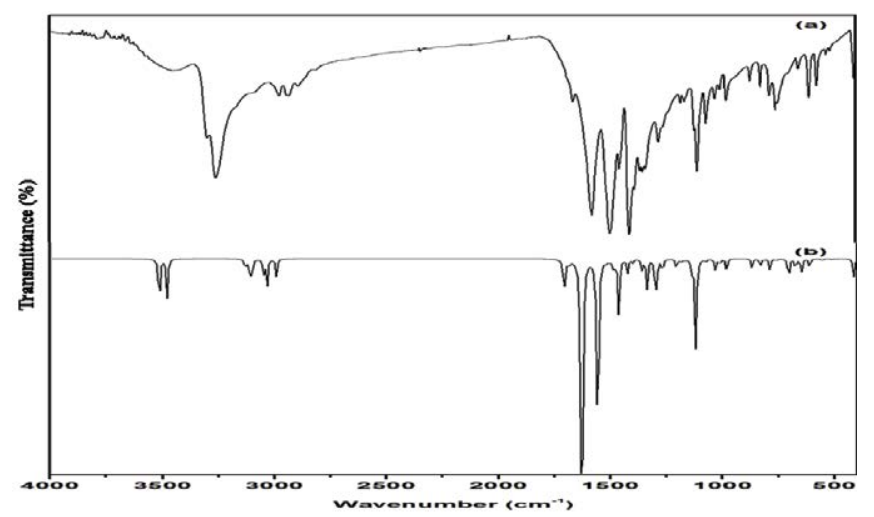

Figure 5: Comparison of observed and calculated infrared spectra of $\mathrm{dmdb}(\mathbf{A})$ Observed and (B) calculated spectra.

\section{Vibrational assignments}

The cemb molecule is a 29 atomic molecule with 81 normal modes of vibration. The observed and calculated IR and Raman spectra of cemb are reproduced in Figures 5 and 6 . The distribution of the normal modes could be divided under the following sections:- (i)

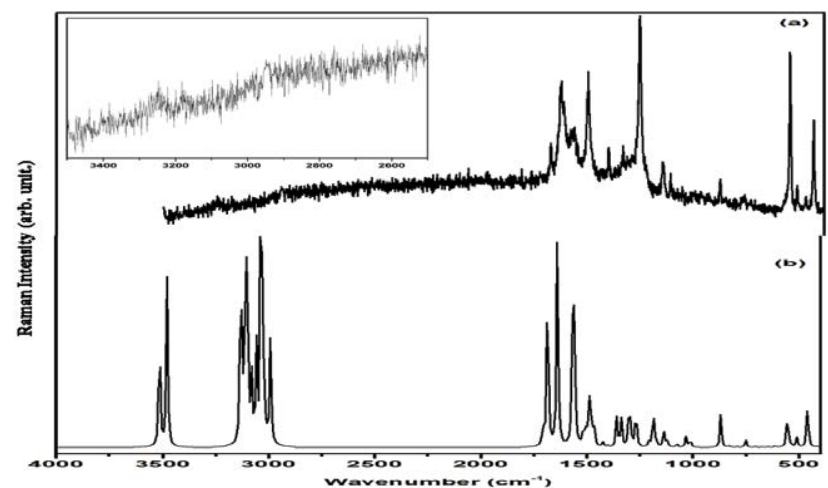

Figure 6: Comparison of observed and calculated Raman spectra of $\mathrm{dmdb}$ (A) Observed and (B) calculated spectra.

$\mathrm{CH}_{3}$ group modes (27 modes) and $\mathrm{CH}_{2}$ group modes (6 modes), (ii) $\mathrm{C}=\mathrm{O}$ modes (6 modes), (iii) Ring modes (12 modes), (iv) $\mathrm{C}-\mathrm{NH}\left(\mathrm{CH}_{3}\right)$ modes (6 modes), (v) $\mathrm{N}-\mathrm{H}$ modes (6 modes), (vi) $\mathrm{N}-\mathrm{CH}_{3}$ modes (6 modes), (vii) $\mathrm{C}-\mathrm{Cl}$ modes (3 modes), (viii) $\mathrm{C}-\mathrm{O}\left(\mathrm{CH}_{2}\right)\left(\mathrm{CH}_{3}\right)$ modes (3 modes), (ix) $\mathrm{O}-\mathrm{CH}_{2}\left(\mathrm{CH}_{3}\right)$ modes ( 3 modes) and (x) $\mathrm{H}_{2} \mathrm{C}-\mathrm{CH}_{3}$ modes (3 modes). The calculated and observed vibrational frequencies with the corresponding PEDs and assignments for the same molecule are collected in Table 4. Assignments for several of the modes agree with the modes of 2,5-diamino-3,6-dichloro-1,4-benzoquinone [46]. In the following we discuss some additional modes briefly.

$\mathrm{CH}_{3}$ group modes (27 modes) and $\mathrm{CH}_{2}$ group modes (6 modes): The ring $\mathrm{CH}$ stretching vibrations fall in the region $3000-3100 \mathrm{~cm}^{-1}$ [47-51]. The symmetric $\mathrm{CH}_{3}$ stretching modes of the $\mathrm{CH}_{3}$ groups, the $\mathrm{CH}_{2}$ symmetric stretching group modes and the antisymmetric $\mathrm{CH}_{3}$ stretching modes of the ${ }^{18} \mathrm{CH}_{3}{ }^{*}$ and ${ }^{14} \mathrm{CH}_{3}$ groups are purely localized modes whereas the antisymmetric stretching modes of the ${ }^{26} \mathrm{CH}_{3}$ and ${ }^{23} \mathrm{CH}_{2}$ groups couple strongly. The assignments for these modes could be seen in Table 4. The antisymmetric deformation modes $\left({ }_{\mathrm{as}}\right)$ of the ${ }^{26} \mathrm{CH}_{3}$, ${ }^{18} \mathrm{CH}_{3}$ and ${ }^{14} \mathrm{CH}_{3}$ groups are assigned at the frequencies $1442\left(v_{56}\right)$ and $1460\left(v_{59}\right), 1444\left(v_{57}\right)$ and $1466\left(v_{60}\right)$ and $1448\left(v_{58}\right)$ and $1467\left(v_{61}\right) \mathrm{cm}^{-1}$. The frequencies $1362\left(v_{52}\right), 1442\left(v_{54}\right)$ and $1427\left(v_{55}\right) \mathrm{cm}^{-1}$ correspond to the symmetric deformation modes $\left({ }_{\mathrm{s}}\right)$ of the groups ${ }^{26} \mathrm{CH}_{3},{ }^{14} \mathrm{CH}_{3}$ and ${ }^{18} \mathrm{CH}_{3}$ respectively. Out of the two rocking modes of the ${ }^{14} \mathrm{CH}_{3}$ group the perpendicular rocking is calculated to have magnitude $1107\left(v_{42}\right)$ $\mathrm{cm}^{-1}$ while the parallel rocking mode has magnitude $1154\left(v_{45}\right) \mathrm{cm}^{-1}$. Similarly, the ${ }^{18} \mathrm{CH}_{3}$ and ${ }^{26} \mathrm{CH}_{3}$ groups also have two rocking modes at the frequencies $1173\left(v_{46}\right)$ and $1111\left(v_{43}\right)$ and $810\left(v_{33}\right)$ and $1103\left(v_{41}\right)$ $\mathrm{cm}^{-1}$. These rocking modes are coupled with each other and many other modes. The torsional modes $\tau\left({ }^{18} \mathrm{CH}_{3}\right), \tau\left({ }^{14} \mathrm{CH}_{3}\right)$ and $\tau\left({ }^{26} \mathrm{CH}_{3}\right)$ are assigned at $107\left(v_{6}\right) 171\left(v_{10}\right)$ and $232\left(v_{14}\right)$ respectively.

Out of the rest four modes of the $\mathrm{CH}_{2}$ group the scissoring mode is calculated to have magnitude $1476\left(v_{62}\right) \mathrm{cm}^{-1}$, the wagging mode is found at $1384\left({ }_{53}\right) \mathrm{cm}^{-1}$ while the twisting and the rocking modes have the magnitudes $1268\left(v_{49}\right)$ and $1141\left(v_{44}\right) \mathrm{cm}^{-1}$.

$\mathrm{C}=\mathbf{O}$ modes (6 modes): The two carbonyl stretching modes couple strongly with the ring stretching modes to give rise to the computed frequencies $1661,1639,1595$ and $1584 \mathrm{~cm}^{-1}$; the former two frequencies being assigned to the ${ }^{\circledR}$ opc $v(\mathrm{C}=\mathrm{O})$ and ${ }^{\circledR}$ ipc $v(\mathrm{C}=\mathrm{O})$ modes whereas the latter two frequencies are assigned to ring stretching modes. The two $\gamma(\mathrm{C}=\mathrm{O})$ modes are assigned at 757 and $745 \mathrm{~cm}^{-1}$ whereas the 2 $\beta(\mathrm{C}=\mathrm{O})$ modes are assigned as the frequencies 412 and $321 \mathrm{~cm}^{-1}$ in 
Citation: Gautam BPS, Dani RK, Prasad RL, Srivastava M, Yadav RA, et al. (2015) Synthesis, Characterization, Single Crystal Structural Studies, Antibacterial Activity and DFT Investigations of 2-Chloro-5-Ethoxy-3,6-Bis(Methylamino)-1,4-Benzoquinone. Pharm Anal Acta 6: 418. doi:10.4172/21532435.1000418

Page 5 of 10

\begin{tabular}{|c|c|c|c|c|}
\hline \multirow{3}{*}{ S. No. } & \multicolumn{3}{|l|}{$\mathbb{R}$} & \multirow{3}{*}{ Vibrational assignment (\%PED) } \\
\hline & \multicolumn{2}{|c|}{$\begin{array}{l}\text { Theoretical wave } \\
\text { number }\end{array}$} & \multirow[t]{2}{*}{$\begin{array}{l}\text { Experimental wave } \\
\text { number }\end{array}$} & \\
\hline & Unscaled & Scaled $^{\star}$ & & \\
\hline 1 & 31 & 31 & - & $\delta\left(\mathrm{O}_{22}-\mathrm{C}_{23}\right)[59]+\square(\mathrm{R})[14]+\mathrm{\gamma}\left(\mathrm{C}_{1}-\mathrm{N}_{8}\right)[9]+\mathrm{\gamma}\left(\mathrm{C}_{4}-\mathrm{N}_{7}\right)[9]$ \\
\hline 2 & 37 & 36 & - & $\mathrm{T}\left(\mathrm{O}_{22}-\mathrm{C}_{23}\right)[31]-\delta\left(\mathrm{C}_{23}-\mathrm{C}_{26}\right)[19]+\mathrm{T}\left(\mathrm{C}_{1}-\mathrm{N}_{8}\right)[8]+\beta\left(\mathrm{C}_{3}-\mathrm{O}_{22}\right)[8]+\square(\mathrm{R})[8]$ \\
\hline 3 & 56 & 55 & - & $\delta\left(\mathrm{O}_{22}-\mathrm{C}_{23}\right)[70]+\mathrm{T}\left(\mathrm{C}_{1}-\mathrm{N}_{8}\right)[9]+\square(\mathrm{R})[7]$ \\
\hline 4 & 63 & 62 & - & $\square(\mathrm{R})[35]+\mathrm{T}\left(\mathrm{C}_{4}-\mathrm{N}_{7}\right)[15]+\delta\left(\mathrm{O}_{22}-\mathrm{C}_{23}\right)[17]$ \\
\hline 5 & 82 & 81 & - & $\mathrm{T}\left(\mathrm{C}_{3}-\mathrm{O}_{22}\right)[25]+\delta\left(\mathrm{C}_{23}-\mathrm{C}_{26}\right)[22]+\mathrm{Y}\left(\mathrm{C}_{3}-\mathrm{O}_{22}\right)[21]+\delta\left(\mathrm{O}_{22}-\mathrm{C}_{23}\right)[10]+\square(\mathrm{R})[9]$ \\
\hline 6 & 108 & 107 & - & $\mathrm{V}\left(\mathrm{N}_{7}-\mathrm{H}_{12}\right)[27]+\mathrm{T}\left(\mathrm{N}_{7}-\mathrm{C}_{18}\right)[17]+\square(\mathrm{R})[11]$ \\
\hline 7 & 129 & 128 & - & $\mathrm{V}\left(\mathrm{N}_{8}-\mathrm{H}_{13}\right)[32]+\square(\mathrm{R})[27]+\mathrm{T}\left(\mathrm{C}_{1}-\mathrm{N}_{8}\right)[13]+\mathrm{T}\left(\mathrm{N}_{8}-\mathrm{C}_{14}\right)[10]+\square(\mathrm{R})[9]$ \\
\hline 8 & 147 & 146 & - & $\mathrm{V}\left(\mathrm{C}_{4}-\mathrm{N}_{7}\right)[38]+\square(\mathrm{R})[22]+\mathrm{T}\left(\mathrm{C}_{4}-\mathrm{N}_{7}\right)[9]+\mathrm{T}\left(\mathrm{N}_{7}-\mathrm{C}_{18}\right)[8]$ \\
\hline 9 & 168 & 167 & - & $\mathrm{V}\left(\mathrm{C}_{3}-\mathrm{O}_{22}\right)[38]+\square(\mathrm{R})[17]+\mathrm{\gamma}\left(\mathrm{C}_{4}-\mathrm{N}_{7}\right)[17]+\delta\left(\mathrm{O}_{22}-\mathrm{C}_{23}\right)[8]$ \\
\hline 10 & 173 & 171 & - & $T\left(N_{8}-C_{14}\right)[60]+\square(R)[10]+T\left(C_{1}-N_{8}\right)[9]+\square(R)[9]$ \\
\hline 11 & 190 & 189 & - & $\beta\left(\mathrm{C}_{4}-\mathrm{N}_{7}\right)[27]+\beta\left(\mathrm{C}_{6}-\mathrm{Cl}_{9}\right)[20]+\delta\left(\mathrm{N}_{8}-\mathrm{H}_{13}\right)[10]+\beta\left(\mathrm{C}_{1}-\mathrm{N}_{8}\right)[10]+\delta\left(\mathrm{N}_{7}-\mathrm{H}_{12}\right)[9]$ \\
\hline 12 & 220 & 218 & - & $\mathrm{V}\left(\mathrm{C}_{6}-\mathrm{Cl}_{9}\right)[44]+\square(\mathrm{R})[12]+\square(\mathrm{R})[11]$ \\
\hline 13 & 224 & 222 & - & $\beta\left(C_{6}-C_{9}\right)[29]+\gamma\left(C_{6}-C_{9}\right)[21]+\beta\left(C_{1}-N_{8}\right)[20]$ \\
\hline 14 & 235 & 233 & - & $\mathrm{T}\left(\mathrm{C}_{23}-\mathrm{C}_{26}\right)[56]+\beta\left(\mathrm{C}_{6}-\mathrm{Cl}_{9}\right)[9]$ \\
\hline 15 & 259 & 257 & - & $\mathrm{T}\left(\mathrm{C}_{23}-\mathrm{C}_{26}\right)[16]+\beta\left(\mathrm{C}_{6}-\mathrm{Cl}_{9}\right)[13]+\delta\left(\mathrm{N}_{8}-\mathrm{C}_{14}\right)[10]+\mathrm{v}\left(\mathrm{C}_{4}-\mathrm{C}_{5}\right)[7]$ \\
\hline 16 & 271 & 269 & - & $\alpha(R)[22]-\delta\left(N_{7}-C_{18}\right)[11]-\delta\left(N_{8}-H_{13}\right)[11]-\beta\left(C_{6}-C l_{9}\right)[10]$ \\
\hline 17 & 324 & 321 & - & $\mathrm{V}\left(\mathrm{C}_{3}-\mathrm{O}_{22}\right)[28]-\beta\left(\mathrm{C}_{5}-\mathrm{O}_{10}\right)[15]-\beta\left(\mathrm{C}_{2}-\mathrm{O}_{11}\right)[15]+\alpha(\mathrm{R})[14]-\delta\left(\mathrm{N}_{8}-\mathrm{H}_{13}\right)[10]$ \\
\hline 18 & 335 & 332 & - & $\beta\left(\mathrm{C}_{3}-\mathrm{O}_{22}\right)[45]-\mathrm{V}\left(\mathrm{C}_{3}-\mathrm{O}_{22}\right)[13]$ \\
\hline 19 & 356 & 353 & - & $\delta\left(C_{23}-C_{26}\right)[26]+\gamma\left(C_{3}-O_{22}\right)[17]+\delta\left(O_{22}-C_{23}\right)[17]-v\left(C_{6}-C_{9}\right)[10]-\alpha(R)[7]$ \\
\hline 20 & 379 & 376 & - & $\mathrm{v}\left(\mathrm{C}_{3}-\mathrm{O}_{22}\right)[34]+\delta\left(\mathrm{O}_{22}-\mathrm{C}_{23}\right)[24]-\mathrm{v}\left(\mathrm{C}_{6}-\mathrm{Cl}_{9}\right)[7]$ \\
\hline 21 & 416 & 412 & - & $\beta\left(\mathrm{C}_{5}-\mathrm{O}_{10}\right)[21]-\beta\left(\mathrm{C}_{2}-\mathrm{O}_{11}\right)[20]+\delta\left(\mathrm{N}_{7}-\mathrm{H}_{12}\right)[9]$ \\
\hline 22 & 461 & 457 & - & $\alpha(R)[35]-\delta\left(N_{7}-H_{12}\right)[10]-\delta\left(N_{8}-H_{13}\right)[9]-\alpha(R)[8]-\beta\left(C_{2}-O_{11}\right)[7]$ \\
\hline 23 & 512 & 507 & - & $\mathrm{V}\left(\mathrm{C}_{4}-\mathrm{N}_{7}\right)[15]-\delta\left(\mathrm{O}_{22}-\mathrm{C}_{23}\right)[12]+\delta\left(\mathrm{N}_{8}-\mathrm{H}_{13}\right)[10]+\alpha(\mathrm{R})[9]-\mathrm{V}\left(\mathrm{C}_{3}-\mathrm{O}_{22}\right)[7]$ \\
\hline 24 & 538 & 533 & - & $Y\left(C_{4}-N_{7}\right)[20]+Y\left(C_{1}-N_{8}\right)[20]+\delta\left(O_{22}-C_{23}\right)[15]+Y\left(C_{2}-O_{11}\right)[10]+\square(R)[10]$ \\
\hline 25 & 557 & 551 & 569 & $\delta\left(\mathrm{N}_{7}-\mathrm{H}_{12}\right)[15]+\beta\left(\mathrm{C}_{2}-\mathrm{O}_{11}\right)[11]-\mathrm{v}\left(\mathrm{C}_{2}-\mathrm{C}_{3}\right)[11]-\mathrm{v}\left(\mathrm{C}_{3}-\mathrm{C}_{4}\right)[8]-\mathrm{v}\left(\mathrm{C}_{6}-\mathrm{Cl}_{9}\right)[8]$ \\
\hline 26 & 613 & 608 & - & $\mathrm{\gamma}\left(\mathrm{C}_{3}-\mathrm{O}_{22}\right)[29]-\square(\mathrm{R})[20]-\delta\left(\mathrm{O}_{22}-\mathrm{C}_{23}\right)[10]+\delta\left(\mathrm{C}_{23}-\mathrm{C}_{26}\right)[26]+\mathrm{\gamma}\left(\mathrm{C}_{4}-\mathrm{N}_{7}\right)[7]-\mathrm{\gamma}\left(\mathrm{C}_{1}-\mathrm{N}_{8}\right)[7]$ \\
\hline 27 & 650 & 644 & 641 & $\gamma\left(C_{3}-O_{22}\right)[21]-\alpha(R)[12]-\gamma\left(N_{7}-H_{12}\right)[10]-\gamma\left(C_{1}-N_{8}\right)[8]$ \\
\hline 28 & 677 & 670 & - & $\mathrm{V}\left(\mathrm{C}_{3}-\mathrm{O}_{22}\right)[36]+\mathrm{T}\left(\mathrm{C}_{4}-\mathrm{N}_{7}\right)[20]+\mathrm{Y}\left(\mathrm{N}_{7}-\mathrm{H}_{12}\right)[13]-\square(\mathrm{R})[8]$ \\
\hline 29 & 707 & 700 & - & $\mathrm{V}\left(\mathrm{N}_{8}-\mathrm{H}_{13}\right)[38]+\mathrm{T}\left(\mathrm{C}_{1}-\mathrm{N}_{8}\right)[36]-\mathrm{V}\left(\mathrm{C}_{4}-\mathrm{C}_{5}\right)[8]$ \\
\hline 30 & 753 & 745 & - & $\square(\mathrm{R})[32]+\mathrm{\gamma}\left(\mathrm{C}_{3}-\mathrm{O}_{22}\right)[30]-\mathrm{\gamma}\left(\mathrm{C}_{2}-\mathrm{O}_{11}\right)[14]+\mathrm{\gamma}\left(\mathrm{C}_{5}-\mathrm{O}_{10}\right)[7]$ \\
\hline 31 & 764 & 757 & 752 & $\mathrm{~V}\left(\mathrm{C}_{5}-\mathrm{O}_{10}\right)[27]+\square(\mathrm{R})[21]+\mathrm{Y}\left(\mathrm{C}_{2}-\mathrm{O}_{11}\right)[17]-\mathrm{Y}\left(\mathrm{C}_{4}-\mathrm{N}_{7}\right)[14]-\square(\mathrm{R})[11]$ \\
\hline 32 & 791 & 784 & - & $\beta\left(\mathrm{C}_{5}-\mathrm{O}_{10}\right)[24]+\mathrm{v}\left(\mathrm{C}_{6}-\mathrm{Cl}_{9}\right)[20]+\beta\left(\mathrm{C}_{4}-\mathrm{N}_{7}\right)[8]+\beta\left(\mathrm{C}_{3}-\mathrm{O}_{22}\right)[7]-\mathrm{v}\left(\mathrm{N}_{7}-\mathrm{C}_{18}\right)[7]$ \\
\hline 33 & 818 & 811 & - & $\delta\left(\mathrm{C}_{26}-\mathrm{H}_{27}\right)[37]-\delta\left(\mathrm{C}_{23}-\mathrm{H}_{25}\right)[37]+\delta\left(\mathrm{C}_{23}-\mathrm{H}_{25}\right)[11]$ \\
\hline 34 & 834 & 826 & - & $\beta\left(C_{1}-N_{8}\right)[18]+\beta\left(C_{2}-O_{11}\right)[13]+\beta\left(C_{3}-O_{22}\right)[9]-v\left(C_{6}-C_{9}\right)[9]$ \\
\hline 35 & 871 & 863 & 890 & $\mathrm{v}\left(\mathrm{O}_{22}-\mathrm{C}_{23}\right)[34]+\delta\left(\mathrm{C}_{26}-\mathrm{H}_{27}\right)[22]+\mathrm{v}\left(\mathrm{C}_{3}-\mathrm{O}_{22}\right)[11]$ \\
\hline 36 & 983 & 974 & - & $v\left(N_{8}-C_{14}\right)[14]+v\left(C_{1}-C_{2}\right)[14]+\beta\left(C_{1}-N_{8}\right)[8]-\beta\left(C_{3}-O_{22}\right)[7]$ \\
\hline 37 & 1012 & 1002 & - & $v\left(C_{23}-C_{26}\right)[17]+\beta\left(C_{4}-N_{7}\right)[9]+v\left(N_{8}-C_{14}\right)[9]+v\left(C_{4}-C_{5}\right)[8]-\delta\left(C_{26}-H_{27}\right)[8]+\beta\left(C_{3}-O_{22}\right)[7]+v\left(N_{7}-C_{18}\right)[7]$ \\
\hline 38 & 1032 & 1022 & - & $\mathrm{v}\left(\mathrm{C}_{23}-\mathrm{C}_{26}\right)[22]-\mathrm{v}\left(\mathrm{N}_{7}-\mathrm{C}_{18}\right)[21]-\mathrm{v}\left(\mathrm{O}_{22}-\mathrm{C}_{23}\right)[12]-\mathrm{v}\left(\mathrm{N}_{8}-\mathrm{C}_{14}\right)[11]$ \\
\hline 39 & 1075 & 1065 & 965 & $\mathrm{v}\left(\mathrm{N}_{7}-\mathrm{C}_{18}\right)[18]-\mathrm{v}\left(\mathrm{O}_{22}-\mathrm{C}_{23}\right)[15]-\mathrm{v}\left(\mathrm{C}_{4}-\mathrm{C}_{5}\right)[12]+\mathrm{v}\left(\mathrm{C}_{23}-\mathrm{C}_{26}\right)[9]-\mathrm{a}(\mathrm{R})[7]$ \\
\hline 40 & 1119 & 1088 & 1128 & $v\left(C_{3}-\mathrm{O}_{22}\right)[19]-v\left(\mathrm{O}_{22}-\mathrm{C}_{23}\right)[17]-\mathrm{v}\left(\mathrm{C}_{1}-\mathrm{C}_{2}\right)[14]+\mathrm{v}\left(\mathrm{N}_{8}-\mathrm{C}_{14}\right)[13]+\delta\left(\mathrm{C}_{26}-\mathrm{H}_{27}\right)[7]$ \\
\hline 41 & 1134 & 1103 & - & $\delta\left(\mathrm{C}_{26}-\mathrm{H}_{27}\right)[41]-\mathrm{v}\left(\mathrm{C}_{23}-\mathrm{C}_{26}\right)[18]$ \\
\hline 42 & 1139 & 1108 & - & $\delta\left(\mathrm{C}_{14}-\mathrm{H}_{15}\right)[67]-\delta\left(\mathrm{C}_{14}-\mathrm{H}_{16}\right)[24]+\delta\left(\mathrm{C}_{14}-\mathrm{H}_{17}\right)[8]$ \\
\hline 43 & 1142 & 1111 & - & $\delta\left(\mathrm{C}_{18}-\mathrm{H}_{19}\right)[77]-\delta\left(\mathrm{C}_{18}-\mathrm{H}_{21}\right)[8]$ \\
\hline 44 & 1173 & 1141 & - & $\delta\left(\mathrm{C}_{23}-\mathrm{H}_{25}\right)[50]+\delta\left(\mathrm{C}_{26}-\mathrm{H}_{27}\right)[29]+\delta\left(\mathrm{C}_{23}-\mathrm{H}_{25}\right)[8]$ \\
\hline 45 & 1187 & 1154 & - & $\delta\left(\mathrm{C}_{14}-\mathrm{H}_{16}\right)[33]+\delta\left(\mathrm{C}_{14}-\mathrm{H}_{15}\right)[11]+\delta\left(\mathrm{C}_{14}-\mathrm{H}_{16}\right)[7]$ \\
\hline 46 & 1206 & 1173 & - & $\delta\left(\mathrm{C}_{18}-\mathrm{H}_{20}\right)[35]-\delta\left(\mathrm{N}_{7}-\mathrm{H}_{12}\right)[9]+\delta\left(\mathrm{C}_{18}-\mathrm{H}_{20}\right)[7]-\mathrm{v}\left(\mathrm{C}_{3}-\mathrm{O}_{22}\right)[7]$ \\
\hline 47 & 1268 & 1234 & - & $v\left(C_{1}-N_{8}\right)[13]+\beta\left(N_{8}-H_{13}\right)[10]-\beta\left(C_{1}-N_{8}\right)[9]+v\left(C_{5}-C_{6}\right)[8]-v\left(N_{8}-C_{14}\right)[7]+\beta\left(C_{5}-O_{10}\right)[7]$ \\
\hline 48 & 1294 & 1259 & - & $\delta\left(\mathrm{C}_{23}-\mathrm{H}_{25}\right)[33]-\mathrm{v}\left(\mathrm{C}_{4}-\mathrm{N}_{7}\right)[14]-\beta\left(\mathrm{N}_{7}-\mathrm{H}_{12}\right)[9]+\mathrm{v}\left(\mathrm{C}_{3}-\mathrm{O}_{22}\right)[8]$ \\
\hline 49 & 1304 & 1269 & - & $\delta\left(\mathrm{C}_{23}-\mathrm{H}_{25}\right)[39]+\mathrm{v}\left(\mathrm{C}_{4}-\mathrm{N}_{7}\right)[13]+\beta\left(\mathrm{N}_{7}-\mathrm{H}_{12}\right)[9]-\mathrm{v}\left(\mathrm{C}_{3}-\mathrm{O}_{22}\right)[9]$ \\
\hline 50 & 1337 & 1301 & 1332 & $v\left(C_{5}-C_{6}\right)[39]-v\left(C_{4}-C_{5}\right)[13]-v\left(C_{1}-N_{8}\right)[11]-v\left(C_{1}-C_{6}\right)[8]+\beta\left(C_{5}-O_{10}\right)[7]$ \\
\hline 51 & 1359 & 1322 & - & $v\left(C_{2}-C_{3}\right)[39]-v\left(C_{3}-O_{22}\right)[10]-v\left(C_{1}-C_{2}\right)[9]+\beta\left(C_{2}-O_{11}\right)[8]$ \\
\hline 52 & 1401 & 1362 & 1405 & $\delta\left(\mathrm{C}_{26}-\mathrm{H}_{27}\right)[60]-\delta\left(\mathrm{C}_{23}-\mathrm{H}_{24}\right)[31]$ \\
\hline 53 & 1423 & 1384 & - & $\delta\left(\mathrm{C}_{23}-\mathrm{H}_{24}\right)[45]+\delta\left(\mathrm{C}_{26}-\mathrm{H}_{27}\right)[31]+\mathrm{v}\left(\mathrm{C}_{23}-\mathrm{C}_{26}\right)[11]$ \\
\hline 54 & 1463 & 1423 & - & $\delta\left(\mathrm{C}_{14}-\mathrm{H}_{15}\right)[72]-\delta\left(\mathrm{C}_{18}-\mathrm{H}_{19}\right)[14]$ \\
\hline 55 & 1468 & 1428 & - & $\delta\left(\mathrm{C}_{18}-\mathrm{H}_{19}\right)[70]+\delta\left(\mathrm{C}_{14}-\mathrm{H}_{15}\right)[16]$ \\
\hline
\end{tabular}


Citation: Gautam BPS, Dani RK, Prasad RL, Srivastava M, Yadav RA, et al. (2015) Synthesis, Characterization, Single Crystal Structural Studies, Antibacterial Activity and DFT Investigations of 2-Chloro-5-Ethoxy-3,6-Bis(Methylamino)-1,4-Benzoquinone. Pharm Anal Acta 6: 418. doi:10.4172/21532435.1000418

Page 6 of 10

\begin{tabular}{|c|c|c|c|c|}
\hline 56 & 1482 & 1442 & - & $\delta\left(\mathrm{C}_{26}-\mathrm{H}_{28}\right)[61]-\delta\left(\mathrm{C}_{26}-\mathrm{H}_{29}\right)[28]-\delta\left(\mathrm{C}_{26}-\mathrm{H}_{27}\right)[8]$ \\
\hline 57 & 1485 & 1444 & - & $\delta\left(\mathrm{C}_{18}-\mathrm{H}_{21}\right)[87]-\delta\left(\mathrm{C}_{18}-\mathrm{H}_{19}\right)[8]$ \\
\hline 58 & 1489 & 1449 & 1470 & $\delta\left(\mathrm{C}_{14}-\mathrm{H}_{17}\right)[91]-\delta\left(\mathrm{C}_{14}-\mathrm{H}_{15}\right)[7]$ \\
\hline 59 & 1501 & 1460 & - & $\delta\left(\mathrm{C}_{26}-\mathrm{H}_{29}\right)[52]+\delta\left(\mathrm{C}_{28}-\mathrm{H}_{28}\right)[23]-\delta\left(\mathrm{C}_{23}-\mathrm{H}_{24}\right)[10]-\delta\left(\mathrm{C}_{26}-\mathrm{H}_{27}\right)[7]$ \\
\hline 60 & 1507 & 1466 & - & $\delta\left(\mathrm{C}_{18}-\mathrm{H}_{20}\right)[66]-\delta\left(\mathrm{C}_{14}-\mathrm{H}_{16}\right)[11]-\delta\left(\mathrm{C}_{18}-\mathrm{H}_{20}\right)[7]$ \\
\hline 61 & 1508 & 1467 & - & $\delta\left(\mathrm{C}_{14}-\mathrm{H}_{16}\right)[72]+\delta\left(\mathrm{C}_{18}-\mathrm{H}_{20}\right)[11]-\delta\left(\mathrm{C}_{14}-\mathrm{H}_{16}\right)[7]$ \\
\hline 62 & 1517 & 1476 & - & $\delta\left(\mathrm{C}_{23}-\mathrm{H}_{24}\right)[86]+\delta\left(\mathrm{C}_{26}-\mathrm{H}_{29}\right)[6]$ \\
\hline 63 & 1557 & 1514 & 1554 & $\beta\left(\mathrm{N}_{8}-\mathrm{H}_{13}\right)[39]-\beta\left(\mathrm{N}_{7}-\mathrm{H}_{12}\right)[13]-\mathrm{v}\left(\mathrm{C}_{2}-\mathrm{O}_{11}\right)[9]$ \\
\hline 64 & 1564 & 1522 & - & $\beta\left(\mathrm{N}_{7}-\mathrm{H}_{12}\right)[35]+\beta\left(\mathrm{N}_{8}-\mathrm{H}_{13}\right)[13]+\mathrm{v}\left(\mathrm{C}_{4}-\mathrm{N}_{7}\right)[8]$ \\
\hline 65 & 1628 & 1584 & - & $v\left(C_{1}-C_{6}\right)[23]-v\left(C_{3}-C_{4}\right)[20]-v\left(C_{2}-O_{11}\right)[11]-v\left(C_{1}-N_{8}\right)[10]$ \\
\hline 66 & 1639 & 1595 & 1602 & $v\left(C_{1}-N_{8}\right)[15]-v\left(C_{3}-C_{4}\right)[14]-v\left(C_{1}-C_{6}\right)[14]-v\left(C_{5}-O_{10}\right)[8]-v\left(C_{2}-O_{11}\right)[8]+v\left(C_{4}-N_{7}\right)[7]$ \\
\hline 67 & 1685 & 1639 & - & $\mathrm{v}\left(\mathrm{C}_{2}-\mathrm{O}_{11}\right)[32]+\mathrm{v}\left(\mathrm{C}_{5}-\mathrm{O}_{10}\right)[29]-\mathrm{v}\left(\mathrm{C}_{3}-\mathrm{C}_{4}\right)[13]+\mathrm{v}\left(\mathrm{C}_{4}-\mathrm{N}_{7}\right)[7]$ \\
\hline 68 & 1707 & 1661 & 1692 & $v\left(C_{5}-O_{10}\right)[36]-v\left(C_{2}-O_{11}\right)[18]+\alpha(R)[9]+v\left(C_{1}-N_{8}\right)[8]-v\left(C_{4}-N_{7}\right)[7]$ \\
\hline 69 & 2992 & 2910 & 2842 & $\mathrm{v}\left(\mathrm{C}_{23}-\mathrm{H}_{24}\right)[94]$ \\
\hline 70 & 3033 & 2950 & 2989 & $\mathrm{v}\left(\mathrm{C}_{18}-\mathrm{H}_{19}\right)[69]+\mathrm{v}\left(\mathrm{C}_{18}-\mathrm{H}_{21}\right)[14]+\mathrm{v}\left(\mathrm{C}_{18}-\mathrm{H}_{20}\right)[11]$ \\
\hline 71 & 3034 & 2951 & 3015 & $\mathrm{v}\left(\mathrm{C}_{26}-\mathrm{H}_{29}\right)[35]+\mathrm{v}\left(\mathrm{C}_{26}-\mathrm{H}_{27}\right)[34]+\mathrm{v}\left(\mathrm{C}_{26}-\mathrm{H}_{28}\right)[26]$ \\
\hline 72 & 3049 & 2966 & 3042 & $v\left(\mathrm{C}_{14}-\mathrm{H}_{16}\right)[40]+\mathrm{v}\left(\mathrm{C}_{14}-\mathrm{H}_{15}\right)[34]+\mathrm{v}\left(\mathrm{C}_{14}-\mathrm{H}_{17}\right)[26]$ \\
\hline 73 & 3081 & 2997 & - & $\mathrm{v}\left(\mathrm{C}_{23}-\mathrm{H}_{25}\right)[71]+\mathrm{v}\left(\mathrm{C}_{26}-\mathrm{H}_{29}\right)[15]$ \\
\hline 74 & 3101 & 3017 & - & $\mathrm{v}\left(\mathrm{C}_{26}-\mathrm{H}_{27}\right)[53]-\mathrm{v}\left(\mathrm{C}_{26}-\mathrm{H}_{29}\right)[23]+\mathrm{v}\left(\mathrm{C}_{23}-\mathrm{H}_{25}\right)[12]-\mathrm{v}\left(\mathrm{C}_{26}-\mathrm{H}_{28}\right)[9]$ \\
\hline 75 & 3102 & 3017 & - & $\mathrm{v}\left(\mathrm{C}_{18}-\mathrm{H}_{21}\right)[39]+\mathrm{v}\left(\mathrm{C}_{18}-\mathrm{H}_{20}\right)[31]-\mathrm{v}\left(\mathrm{C}_{18}-\mathrm{H}_{19}\right)[27]$ \\
\hline 76 & 3110 & 3026 & - & $\mathrm{v}\left(\mathrm{C}_{26}-\mathrm{H}_{28}\right)[59]-\mathrm{v}\left(\mathrm{C}_{26}-\mathrm{H}_{29}\right)[25]+\mathrm{v}\left(\mathrm{C}_{23}-\mathrm{H}_{25}\right)[11]$ \\
\hline 77 & 3114 & 3029 & - & $\mathrm{v}\left(\mathrm{C}_{14}-\mathrm{H}_{16}\right)[52]-\mathrm{v}\left(\mathrm{C}_{14}-\mathrm{H}_{15}\right)[47]$ \\
\hline 78 & 3127 & 3042 & - & $\mathrm{v}\left(\mathrm{C}_{14}-\mathrm{H}_{17}\right)[72]-\mathrm{v}\left(\mathrm{C}_{14}-\mathrm{H}_{15}\right)[19]-\mathrm{v}\left(\mathrm{C}_{14}-\mathrm{H}_{16}\right)[8]$ \\
\hline 79 & 3135 & 3049 & - & $\mathrm{v}\left(\mathrm{C}_{18}-\mathrm{H}_{20}\right)[55]-\mathrm{v}\left(\mathrm{C}_{18}-\mathrm{H}_{21}\right)[44]$ \\
\hline 80 & 3479 & 3385 & 3356 & $\mathrm{v}\left(\mathrm{N}_{8}-\mathrm{H}_{13}\right)[99]$ \\
\hline 81 & 3515 & 3419 & - & $\mathrm{v}\left(\mathrm{N}_{7}-\mathrm{H}_{12}\right)[99]$ \\
\hline
\end{tabular}

The numbers (in bracket) after the modes are the \% potential energy calculated using normal coordinate analysis. The abbreviations are: $v=s t r e t c h i n g, \omega=w a g g i n g$, $T=$ twisting, $\rho=$ rocking, $\sigma=$ scissoring, $\gamma=$ out-of-plane deformation, $\beta=$ =in-plane deformation, $\alpha=$ planar ring deformation, $\varphi=$ non-planar ring deformation, $\delta=$ deformation. The modes in bold letters in the last column are the assigned modes.

*With the scale factor of 0.9906 for calculated wave numbers lower than $1100 \mathrm{~cm}^{-1}$ and the scale factor 0.9728 for larger wave numbers

Table 4: Vibrational assignments of cemb

accordance with the assignment reported in literature [52].

Ring modes (12 modes): In addition to the two ring stretching modes mentioned above (section 5.1.2) the frequencies $1321\left(v_{51}\right)$, $1300\left({ }_{50}\right), 974\left(v_{36}\right)$ and $551\left(v_{25}\right) \mathrm{cm}^{-1}$ have been identified as the ring stretching modes. The lowest frequency mode $551\left(v_{25}\right) \mathrm{cm}^{-1}$ corresponds to the ring breathing mode. Out of the three planar ring deformation frequencies $641\left(v_{27}\right), 507\left({ }_{23}\right)$ and $457\left(v_{22}\right) \mathrm{cm}^{-1}$ the first frequency appears to be due to the trigonal phenyl ring bending mode whereas the latter two correspond to the modes $6(\mathrm{a}, \mathrm{b})$ of the benzene molecule. The three normal modes of vibration corresponding to the non-planar ring deformations are calculated to have magnitudes 608 $\left(v_{26}\right), 146\left({ }_{8}\right)$ and $128\left(v_{7}\right) \mathrm{cm}^{-1}$. All the 12 normal modes of the ring are strongly coupled with the other modes.

$\left(\mathrm{C}-\mathrm{NHCH}_{3}\right)$ modes (6 modes): The frequencies $1233\left({ }_{47}\right)$ and $1258\left(v_{48}\right) \mathrm{cm}^{-1}$ corresponds to $v\left(\mathrm{C}_{1}-\mathrm{NHCH}_{3}\right)$ and $v\left(\mathrm{C}_{4}-\mathrm{NHCH}_{3}\right)$, respectively. The two planar $\mathrm{C}-\mathrm{NHCH}_{3}$ bending mode are found to have widely differing magnitudes $826\left({ }_{34}\right) \mathrm{cm}^{-1}$ and $188\left(v_{11}\right) \mathrm{cm}^{-1}$. The non-planar bending modes are calculated to have frequencies $167\left({ }_{9}\right)$ $\mathrm{cm}^{-1}$ and $31\left(v_{1}\right) \mathrm{cm}^{-1}$.

$\mathrm{N}-\mathrm{H}$ modes (6 modes): The wave numbers $3419\left(v_{81}\right) \mathrm{cm}^{-1}$ and 3384 $\left({ }_{80}\right) \mathrm{cm}^{-1}$ correspond $\mathrm{N}_{7}-\mathrm{H}$ and $\mathrm{N}_{8}-\mathrm{H}$ stretching vibrations. The $\mathrm{N}-\mathrm{H}$ stretching vibrations are pure and highly localized modes. The $\beta\left(\mathrm{N}_{7}-\right.$ $\mathrm{H})$ and $\beta\left(\mathrm{N}_{8}-\mathrm{H}\right)$ modes are calculated to have magnitudes $1521\left(v_{64}\right)$ $\mathrm{cm}^{-1}$ and $1514\left({ }_{63}\right) \mathrm{cm}^{-1}$, respectively. The out-of-plane deformations corresponding to the modes $\gamma\left(\mathrm{N}_{7}-\mathrm{H}\right)$ and $\gamma\left(\mathrm{N}_{8}-\mathrm{H}\right)$ are correlated to the calculated frequencies $670\left({ }_{28}\right) \mathrm{cm}^{-1}$ and $700\left({ }_{29}\right) \mathrm{cm}^{-1}$ respectively.

${ }^{\circledR \mathrm{n}} \mathrm{C}$ means $\mathrm{C}$ atom with number $\mathrm{n} ;{ }^{\circledR} \mathrm{opc}=$ out of phase coupled and ipc $=$ in phase coupled

$\mathrm{N}-\mathrm{CH}_{3}$ modes (6 modes): The stretching modes corresponding to $v\left(\mathrm{~N}_{7}-\mathrm{CH}_{3}\right)$ and $v\left(\mathrm{~N}_{8}-\mathrm{CH}_{3}\right)$ are found at the frequencies $\left.1064{ }_{39}\right) \mathrm{cm}^{-1}$ and $1002\left(v_{37}\right) \mathrm{cm}^{-1}$ respectively. The twisting modes are calculated to have magnitudes $62\left(v_{1}\right)$ and $55\left(v_{3}\right)$ corresponding to the modes $\tau\left(\mathrm{N}_{7}\right.$ $\left.\mathrm{CH}_{3}\right)$ and $\left(\mathrm{N}_{8}-\mathrm{CH}_{3}\right)$, respectively while the angle bending modes are assigned at $268\left(v_{16}\right)$ and $257\left(v_{15}\right)$ wave numbers corresponding to the modes $\alpha\left(\mathrm{C}_{4}-\mathrm{N}_{7}-\mathrm{CH}_{3}\right)$ and $\alpha\left(\mathrm{C}_{1}-\mathrm{N}_{8}-\mathrm{CH}_{3}\right)$.

C-Cl modes (3 modes): In benzene derivatives $\mathrm{C}-\mathrm{Cl}$ stretching is observed in wide spectral range $700-1050 \mathrm{~cm}^{-1}$ [53]. The present calculations place the $\mathrm{C}-\mathrm{Cl}$ stretching mode at frequency $783\left(v_{32}\right) \mathrm{cm}^{-1}$. The modes corresponding to the planar deformation mode $\beta$ (C-Cl) and non-planar deformation mode $\gamma(\mathrm{C}-\mathrm{Cl})$ are at frequencies $222\left(v_{13}\right)$ and $218\left(v_{12}\right) \mathrm{cm}^{-1}$ respectively.

C- $\mathrm{O}\left(\mathrm{CH}_{2}\right)\left(\mathrm{CH}_{3}\right)$ modes (3 modes): The $\mathrm{C}_{3}-\mathrm{O}\left(\mathrm{CH}_{2}\right)\left(\mathrm{CH}_{3}\right)$ bond gives rise to 3 normal modes of vibration as: a $v\left[\mathrm{C}_{3}-\mathrm{O}\left(\mathrm{CH}_{2}\right)\left(\mathrm{CH}_{3}\right)\right]$, a $\beta\left[\mathrm{C}_{3}-\mathrm{O}\left(\mathrm{CH}_{2}\right)\left(\mathrm{CH}_{3}\right)\right]$ and a $\gamma\left[\mathrm{C}_{3}-\mathrm{O}\left(\mathrm{CH}_{2}\right)\left(\mathrm{CH}_{3}\right)\right]$ modes. The wavenumbers $1088\left(v_{40}\right), 332\left(v_{18}\right)$ and $376\left(v_{20}\right)$, correspond to the above three modes respectively.

O- $\mathrm{CH}_{2}\left(\mathrm{CH}_{3}\right)$ modes (3 modes): Three normal modes of vibrations $v\left(\mathrm{O}_{22}-\mathrm{CH}_{2}\left(\mathrm{CH}_{3}\right)\right), \tau\left(\mathrm{O}_{22}-\mathrm{CH}_{2}\left(\mathrm{CH}_{3}\right)\right)$ about $\mathrm{C}_{3}-\mathrm{O}_{22}$ bond and $\alpha\left(\mathrm{C}_{3}-\mathrm{O}_{22}-\right.$ $\left.\mathrm{CH}_{2}\left(\mathrm{CH}_{3}\right)\right)$ arise due to $\mathrm{O}_{22}-\mathrm{CH}_{2}\left(\mathrm{CH}_{3}\right)$ bond. The stretching mode is found at the frequency $862\left({ }_{35}\right) \mathrm{cm}^{-1}$. The twisting mode is calculated to have magnitude $81\left(_{5}\right) \mathrm{cm}^{-1}$ while the angle bending mode is assigned at 
Citation: Gautam BPS, Dani RK, Prasad RL, Srivastava M, Yadav RA, et al. (2015) Synthesis, Characterization, Single Crystal Structural Studies, Antibacterial Activity and DFT Investigations of 2-Chloro-5-Ethoxy-3,6-Bis(Methylamino)-1,4-Benzoquinone. Pharm Anal Acta 6: 418. doi:10.4172/21532435.1000418

Page 7 of 10

$533\left(v_{24}\right)$ wave number.

5.3.10 $\mathrm{C}\left(\mathrm{H}_{2}\right)-\mathrm{C}\left(\mathrm{H}_{3}\right)$ modes (3 modes): Three normal modes of vibrations $v(\mathrm{C}-\mathrm{C}), \tau(\mathrm{C}-\mathrm{C})$ and $\alpha(\mathrm{O}-\mathrm{C}-\mathrm{C})$ arise due to $\mathrm{H}_{2} \mathrm{C}-\mathrm{CH}_{3}$ modes. The stretching mode corresponding to $v\left(\mathrm{C}_{23}-\mathrm{C}_{26}\right)$ is found at the frequency $1022 \mathrm{~cm}^{-1}\left({ }_{38}\right)$. The twisting mode is calculated to have magnitude $36\left(v_{2}\right) \mathrm{cm}^{-1}$ while the angle bending mode is assigned at 352 $\left(v_{19}\right)$ wave number.

\section{Molecular electrostatic potential and contour maps}

The molecular electrostatic potential (MEP) mapped surfaces illustrate the charge distributions of molecules three dimensionally which give clear signature of the interactions of the molecules. The electrostatic potential is used to find the reactive site of a molecule. The MEP mapped surface of the molecules are calculated by DFT/ B3LYP/6-311G (d,p) method at the 0.02 isovalues and 0.004 density values. In color scheme of MEP plot, the red region show atoms with lone pairs or negative electrostatic potentials; the intensity of the color is proportional to the absolute value of the potential energy. The positive electrostatic potentials are shown in blue/yellow color region and characterize the polar hydrogen in $\mathrm{O}-\mathrm{H}$ bonds. The Green areas cover parts of the molecule where electrostatic potentials are close to zero ( $\mathrm{C}-\mathrm{C}, \mathrm{C}-\mathrm{N}$ and $\mathrm{C}-\mathrm{S}$ bonds). The MEP mapped surface of the cemb is shown in Figure 4(B).

Molecular electrostatic potential and electrostatic potential are the useful quantities to illustrate the charge distributions of molecules are used to visualize variably charged regions of a molecule. Therefore, the charge distributions can give the information about how the molecules interact with another molecule. At any given point $r(x, y$, $\mathrm{z}$ ) in the vicinity of a molecule, the molecular electrostatic potential (MEP), $V(r)$ is defined in terms of the interaction energy between the electrical charge generated from the molecule electrons and nuclei and a positive test charge (a proton) located at $r$. The molecular electrostatic potential is related to the electronic density and a very useful descriptor for determining sites for electrophilic attack and nucleophilic reactions as well as hydrogen-bonding interactions [54]. In Figure $4 \mathrm{~b}$ and $4 \mathrm{c}$, whereas electrophilic reactivity is presented by the negative (red) regions, nucleophilic reactivity is shown by the positive (blue) regions of MEP. The red region is localized on the vicinity of oxygen and reflects the most electronegative region (excess negative charge). On the other hand, the nucleophilic reactivity of the molecules is localized on the hydrogen atoms. In this respect, the compound (cemb) is found to be useful to both bonds metallically and interact intermolecularly. Based on these results, it can be ascertained that how the change of the chlorine group affects the molecular properties of the compound. One can see from the Figure $4 \mathrm{~b}$ that the blue color on methyl amine is found to be the predominant because of the positive charge distribution on the molecule. Moreover, according to the ESP Figure 4c, the most negative ESP is spread over the oxygen and chlorine atoms, indicating the delocalization of electrons over the oxygen and chlorine atoms of the molecule which also reveal extended conjugation of the benzene ring with these groups [55]. Furthermore, the total charge density contour map is given in the Figure 4d. A contour plot is a two-dimensional XY plot of a three-dimensional XYZ surface showing lines where the surface intersects planes of constant elevation $(\mathrm{Z})$. The contour maps are used to show lines of constant density or brightness, such as electrostatic potentials and are drawn in the molecular plane. The electron rich red lines are around oxygen and nitrogen whereas electron deficient region are shown by greenish-yellow lines. The contour maps are also calculated by same basis sets at 0.004 density values at same level of calculations of the MEP mapped surface of the molecules [56].

\section{Frontier molecular orbital analysis}

The analysis of the wave function indicates that the electron absorption corresponds to the transition from the ground to the first excited state and is mainly described by one-electron excitation from the HOMO to LUMO. Both the HOMO and LUMO are the main orbitals taking part in the chemical reaction. The HOMO energy characterizes the capability of electron donating; The LUMO characterizes the capability of electron accepting [57]. The HOMO and LUMO, calculated at the B3LYP/6-311G $(\mathrm{d}, \mathrm{p})$ level can be seen in Figure 7 for the gas phase. The positive phases are marked as red and the negative ones as green. Figure 7 shows distribution and energy levels of the HOMO and LUMO orbitals. For the HOMO of cemb, electron density are localized on all atom except on methyl of ethoxy group with $\mathrm{E}_{\text {номо }}=-0.21294$ a.u. For the LUMO, electron density are localized on the entire atom except on ethoxy group with $\mathrm{E}_{\mathrm{LMO}}=-0.10412$. The value of the energy separation between the HOMO and LUMO is 0.10882 a.u $(2.961068 \mathrm{eV})$. In the contour map, the lines with red colors are regarded as the negative phase and the lines with green colors are the positive phase. The contour HOMO-LUMO diagram is in accordance with the frontier orbital. The frontier orbital gap helps to characterize the chemical reactivity, optical polarizability and chemical hardnesssoftness of a molecule [58].The small HOMO-LUMO energy gap means low excitation energy for the compound, a good stability and a low chemical hardness. The molecules having a large energy gap are known as hard, and molecules having a small energy gap are known as soft molecules. The soft molecules are more polarizable than the hard ones, because they need small energy for excitation. The hardness value of a molecule can be determined by the formula [59]

$$
\eta=\left\{-\mathrm{E}_{\text {номо }}+\mathrm{E}_{\text {LUMO }}\right\} / 2
$$

where $\mathrm{E}_{\text {номо }}$ and $\mathrm{E}_{\mathrm{LUMO}}$ are the energies of the HOMO and LUMO molecular orbitals. The value of $\eta$ of cemb, 0.05441 a.u $(1.480534 \mathrm{eV})$, indicates for the compound should be a soft material.

\section{Global reactivity descriptors}

On the basis of Koopman's theorem, global reactivity descriptors [60] are calculated using the energies of Frontier molecular orbitals $E_{\text {номо }}, E_{\text {LUмо }}$ and given by Equations (a)-(e).

$$
\begin{aligned}
& \chi=-\left(E_{\text {номо }}+E_{\text {LUмо }}\right) / 2=(I+A) / 2(\mathrm{a}) \\
& \mu=-\chi=\left(E_{\text {номо }}+E_{\text {LUMO }}\right) / 2=-(I+A) / 2(\mathrm{~b})
\end{aligned}
$$

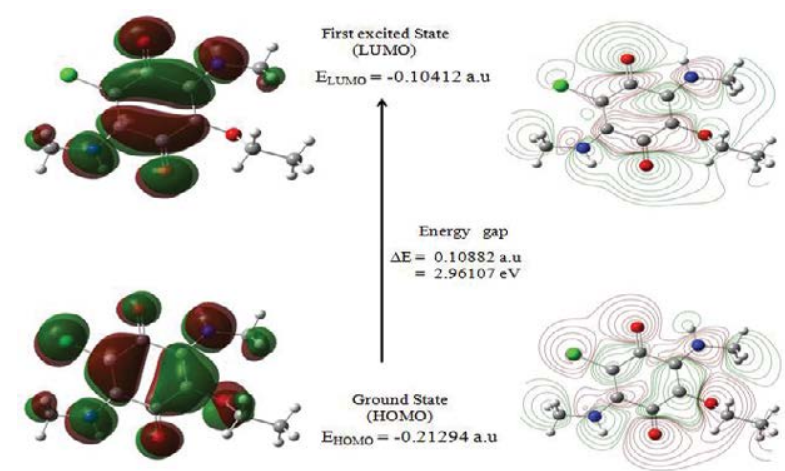

Figure 7: Ground state iso-density surface plots for molecular orbitals of cemb. 
Citation: Gautam BPS, Dani RK, Prasad RL, Srivastava M, Yadav RA, et al. (2015) Synthesis, Characterization, Single Crystal Structural Studies, Antibacterial Activity and DFT Investigations of 2-Chloro-5-Ethoxy-3,6-Bis(Methylamino)-1,4-Benzoquinone. Pharm Anal Acta 6: 418. doi:10.4172/21532435.1000418

$$
\eta=\left(-E_{\mathrm{HOMO}}+E_{\mathrm{LUMO}}\right) / 2=(I-A) / 2(\mathrm{c})
$$

where $A$ and $I$ are the ionization potential and electron affinity of the compounds respectively. Electron affinity refers to the capability of a ligand to accept precisely one electron from a donor. However, in many kinds of bonding viz., covalent hydrogen bonding, and partial charge transfer occurs. Softness $(S)$ is the reciprocal of hardness and is a property of compound that measures the extent of chemical reactivity.

$$
S=1 / \eta(\mathrm{d})
$$

Recently Parr et al. [61] have defined a new descriptor to quantity the global electrophilic power of the compound as electrophilicity index $(\omega)$, which defines a quantitative classification of the global electrophilic nature of a compound. Parr et al. have proposed electrophilicity index $(\omega \mathrm{x})$ as a measure of energy lowering due to maximal electron flow between donor and acceptor. They defined electrophilicity index $(\omega)$ as follows

$$
\omega=\mu^{2} \mathrm{~S}=\mu^{2} / \eta(\mathrm{e})
$$

The energies of frontier molecular orbitals $\left(E_{\text {HOMO }}, E_{\mathrm{LUMO}}\right)$, energy band gap $\left(E_{\text {Hомо }}, E_{\text {LUMO }}\right)$, electro negativity $(\chi)$, ionization potential $(A)$, electron affinity $(I)$, chemical potential $(\mu)$, global hardness $(\eta)$, global softness $(S)$ and global electrophilicity index $(\omega)$ for cemb are listed in Table 5.

\section{In-vitro antibacterial activity}

Six bacterial pathogens Salmonella typhi, Escherichia coli, Shigella flexneri, Staphylococcus aureus, Aeromonas hydrophila and Enterococcus faecalis were used to test the antibacterial activity of the cemb. The activity of cemb and commercial antibacterial drugs streptomycin sulphate and chloramphenicol were tested against the bacterial pathogens and the inhibition zones $(\mathrm{cm})$ of the compounds were presented in the Table 6 . The test results of the compound have also been compared with those of the standard drugs and results show significant activity of the compound against $S$. aureus than all other test pathogens. The cemb displays higher zone of inhibition $(3.5 \mathrm{~cm})$ against S. aureus at $100 \mu \mathrm{g} /$ disc and does not display any zone of inhibition against Aeromonas hydrophila and E.coli at all concentration. A clearing zone around the disc indicates the inhibitory activity of the compound on the organism (Figure 8a-8d). The results are given in Table 6 which clearly indicate that the inhibitions by the compound is lower as compared to the standard drugs and activity increases with increasing concentration of the compound (since concentration plays a vital role in increasing the degree of inhibition) which is almost comparable to the size of the reference antibiotics. 1,4-Benzoquinones is a crossconjugated enediones, have ability to accept one electron initially, forming the semiquinone anion radical, followed by a further electron to give the dianion, is the dominant feature of quinone chemistry. It is this reversible reduction process that accounts for the biological activity [62]. Apart from this, other factors such as solubility, conductivity and intermolecular interaction may also be the possible reasons for activity. Furthermore, free HNCCO moiety increases the delocalization of $\pi$-electrons over the molecule of cemb and enhances the lipophilicity

\begin{tabular}{|l|l|}
\hline Molecular properties & Values in eV \\
\hline Electronegativity $(\mathrm{X})$ & 0.317 \\
\hline Chemical potential $(\mu)$ & -0.317 \\
\hline Global hardness $(\eta)$ & 0.545 \\
\hline Global softness $(\mathrm{s})$ & 1.835 \\
\hline Electrophilicity Index $(\omega)$ & -0.184 \\
\hline
\end{tabular}

Table 5: Reactivity parameters of cemb.
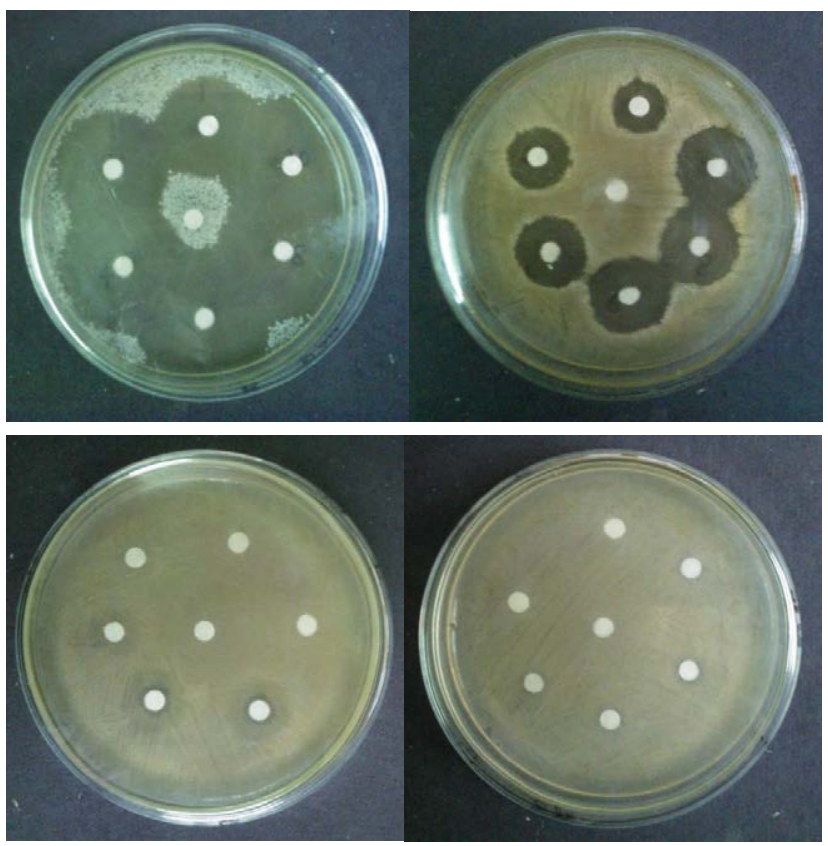

Figure 8: Antibacterial activity of cemb (A) Inhibition zone (IZ) of cemb against $S$. aureus (Center disc=DMSO, -ve control) (B) IZ against $S$. flexneri

\begin{tabular}{|c|c|c|c|c|}
\hline \multirow[b]{2}{*}{ Test pathogen } & \multirow{2}{*}{$\begin{array}{l}\text { Concentration } \\
\text { of compound } \\
(\mu \mathrm{g} / \mathrm{disc})\end{array}$} & \multirow{2}{*}{$\begin{array}{l}\text { Compound } \\
\text { (cemb) }\end{array}$} & \multicolumn{2}{|l|}{ Standard drug } \\
\hline & & & $\begin{array}{l}\text { Streptomycin } \\
\text { sulphate }\end{array}$ & Chloramphenicol \\
\hline \multirow{6}{*}{$\begin{array}{l}\text { Salmonella } \\
\text { typhi }\end{array}$} & 10 & - & 2.0 & 2.4 \\
\hline & 20 & - & 2.2 & 2.5 \\
\hline & 40 & - & 2.4 & 2.6 \\
\hline & 60 & 0.9 & 2.8 & 2.9 \\
\hline & 80 & 1.2 & 3.1 & 3.2 \\
\hline & 100 & 1.3 & 3.5 & 3.7 \\
\hline \multirow{6}{*}{ Shigella flexneri } & 10 & 0.6 & 2.0 & 2.6 \\
\hline & 20 & 0.8 & 2.4 & 2.9 \\
\hline & 40 & 0.9 & 2.6 & 3.0 \\
\hline & 60 & 1.1 & 3.0 & 3.3 \\
\hline & 80 & 1.3 & 3.3 & 3.5 \\
\hline & 100 & 1.5 & 3.6 & 3.7 \\
\hline \multirow{6}{*}{$\begin{array}{l}\text { Staphylococcus } \\
\text { aureus }\end{array}$} & 10 & 1.8 & 2.0 & 2.2 \\
\hline & 20 & 2.0 & 2.1 & 2.4 \\
\hline & 40 & 2.4 & 2.3 & 2.5 \\
\hline & 60 & 2.7 & 2.6 & 2.8 \\
\hline & 80 & 2.8 & 2.9 & 3.2 \\
\hline & 100 & 3.5 & 3.6 & 3.8 \\
\hline \multirow{6}{*}{$\begin{array}{l}\text { Enterococcus } \\
\text { faecalis }\end{array}$} & 10 & - & 2.2 & 1.6 \\
\hline & 20 & - & 2.3 & 1.9 \\
\hline & 40 & - & 2.5 & 2.0 \\
\hline & 60 & 0.7 & 2.6 & 2.1 \\
\hline & 80 & 0.9 & 3.0 & 2.3 \\
\hline & 100 & 1.2 & 3.2 & 2.5 \\
\hline
\end{tabular}
(C) IZ against of cemb against S. typhi (D) No inhibition of cemb against $E$. coli.

No zone was observed *inhibition zone is the average of the diameter of zone from two sides in $\mathrm{cm}$. (A. Hydrophila, E. coli does not show activity).

Table 6: Antibacterial assay of the cemb and standard drugs against human bacterial pathogens. 
Citation: Gautam BPS, Dani RK, Prasad RL, Srivastava M, Yadav RA, et al. (2015) Synthesis, Characterization, Single Crystal Structural Studies, Antibacterial Activity and DFT Investigations of 2-Chloro-5-Ethoxy-3,6-Bis(Methylamino)-1,4-Benzoquinone. Pharm Anal Acta 6: 418. doi:10.4172/21532435.1000418

Page 9 of 10

which leads to the breakdown of the permeability barrier of the cell and thus retards the normal cell processes. The mode of action may involve the formation of a hydrogen bond through the nitrogen /oxygen atom in the compound with the active centers of the cell constituents, resulting in interference with the normal cell process, i.e., prolonging the lag phase of the growth cycle and increasing the generation time of the organisms so that it takes each organism more time to complete cell division [63].

\section{Conclusions}

This paper reports the synthesis, spectral and crystal structure investigations of a new compound: 1,4-benzoquinone derivative, 2-chloro-5-ethoxy-3,6-bis (methylamino)-1,4-benzoquinone (cemb), formed via condensation reaction. It was found that during the reaction, chlorine group of the compound is replaced with ethoxy group generating a new molecule which is stabilized via weak intermolecular interactions. The results of antibacterial activity shows that the new compound cemb inhibit growth of Gram-positive bacteria. Keeping in view the single molecule approximation, the $\mathrm{X}$-ray crystallographic results are well reproduced by DFT method. From the vibrational analysis it turns out that the ring breathing mode (551 $\left.\mathrm{cm}^{-1}\right)$ and the trigonal ring bending mode $\left(641 \mathrm{~cm}^{-1}\right)$ are considerably reduced as compared to the benzene modes. In the present case one $\gamma(\mathrm{C}=\mathrm{O})$ is found in the range $700-800 \mathrm{~cm}^{-1}$ while the other $\gamma(\mathrm{C}=\mathrm{O})$ and two $\beta(\mathrm{C}=\mathrm{O})$ are found to be in the range $300-400 \mathrm{~cm}^{-1}$ while for 2,5-diamino-3,6-dichloro-1,4-benzoquinone [46] the two $\gamma(\mathrm{C}=\mathrm{O})$ and one $\beta(\mathrm{C}=\mathrm{O})$ were found in the range $700-800 \mathrm{~cm}^{-1}$ and the other $\beta(\mathrm{C}=\mathrm{O})$ in the range $300-400 \mathrm{~cm}^{-1}$. Inspite of the low symmetry $\left(\mathrm{C}_{1}\right)$ of the molecule the modes of the identical groups couple strongly. According to the MEP, the most negative ESP is spread over the oxygen and chlorine atoms, indicating the delocalization of electrons over the oxygen and chlorine atoms of the molecule which also reveal extended conjugation of the benzene ring.

\section{Acknowledgements}

Authors are thankful to the Department of Chemistry, Banaras Hindu University, Varanasi for providing the laboratory facilities. BPSG and MG express their sincere thanks to UGC New Delhi, India [Grant No. F1-17.1/2013-14/RGNF2013-14-SC-UTT-50941/ (SA-III/Website) and F1-17.1/2011-12/RGNF-SCUTT-6352/ (SA-III/Website)] and MS thanks to the Banaras Hindu University for the financial assistance.

\section{References}

1. Thomson RH (1987) Naturally Occurring Quinones; Chapman and Hall; London.

2. Dalton RD (1979) The Alkaloids; Academic Press: New York, 264

3. Morton A (1965) Biochemistry of Quinones; Academic Press: New York, NY.

4. Bentley R, Campbell IM, Patai S (1974) The Chemistry of Quinoid Compounds; John Wiley and Sons: London, 683

5. Nohl H, Jordan W, Youngman RJ (1986) Quinones in biology: Functions in electron transfer and oxygen activation. Adv Free Radical Biol Med 2: 211.

6. Monks TJ, Hanzlik RP, Cohen GM, Ross D, Graham DG (1992)Contemporary issues in toxicology, Toxicol Appl Pharmacol 112: 2

7. Fox MA, Olive S (1979) Photooxidation of anthracene on atmospheric particulate matter. Science 205: 582-583.

8. Pryor WA, Hales BJ, Premovic PI, Church DF (1983) The radicals in cigarette tar: Their nature and suggested physiological implications. Science 220: 425427 .

9. Lin AJ, Coby LA, Sartorelli AC (1974) Quinones as anticancer agents: Potential bioreductive alkylating agents. Cancer Chemother Rep 24: 23-25.

10. Hoover E, Dag AR (1954) Preparation of some imidazole derivatives of 1,4-naphthoquinone. J Am Chem Soc 76: 4148-4152.

11. Torgeson DC (1968) Fungicides: An Advanced Treatise. Science 161: 155-156.

12. Clark NG (1985) The fungicidal activity of substituted 1, 4-naphthoquinones Part III: Amino, anilino and acylamino derivatives. Pestic Sci 16: 23.

13. Howland JL (1963) The reversibility of inhibition by 2-heptyl-4-hydroxyquinolinen-oxide and 2-hydroxy-3 (3-methylbutyl)-1, 4-naphthoquinone of succinate oxidation. Biochim Biophys Acta 73: 665.

14. Patai S, Rappaport Z (1988) The Chemistry of the Quinonoid Compounds, John Wiley and Sons Ltd., New York.

15. Thomson RH (1997) Naturally Occurring Quinones IV. Recent Advances, Blackie Academic and Professional, London

16. Hartley JA, Reszka K, Lown JW (1988) Photosensitization by Antitumor Agents-7. Correlation between Anthracenedione-Photosensitized DNA Damage, Nadh Oxidation and Oxygen Consumption Following Visible Light Illumination. Photochem Photobiol 48: 19.

17. Koyama J (2006) Anti-infective quinone derivatives of recent patents. Recent Pat Antiinfect Drug Discov 1: 113-125.

18. Gupta SP (1994) Quantitative structure-activity relationship studies on anticancer drugs. Chem Rev 94: 1507-1551.

19. Silva AJM, Netto CD, Pacienza-Lima W, Torres-Santos EC, Rossi-Bergmann B (2009) Antitumoral, antileishmanial and antimalarial activity of pentacyclic 1,4-naphthoquinone derivatives. J Braz Chem Soc 20 176-182.

20. Amaro AR, Oakley GG, Bauer U, Spielmann HP, Robertson LW (1996) Metabolic activation of PCBs to quinones: Reactivity toward nitrogen and sulfur nucleophiles and influence of superoxide dismutase. Chem Res Toxicol 9: 623629.

21. PJ O'brien (1991) Molecular mechanisms of quinone cytotoxicity. Chem Biol Interact 80: 1.

22. Lin TS, Zhu LY, Xu SP, Divo AA, Sartorelli AC (1991) Synthesis and antimalarial activity of 2-aziridinyl- and 2,3-bis(aziridinyl)-1,4-naphthoquinonyl sulfonate and acylate derivatives. J Med Chem 34: 1634-1639.

23. Lin AJ, Lillis BJ, Sartorelli AC (1975) Potential bioreductive alkylating agents. 5. Antineoplastic activity of quinoline-5,8-diones, naphthazarins, and naphthoquinones. J Med Chem 18: 917-921

24. Dowd P, Zheng ZB (1995) On the mechanism of the anticlotting action of vitamin E quinone. Proc Natl Acad Sci U S A 92: 8171-8175.

25. Gonzalez-Ibarra M, Farfan N, Trejo C, Uribe S, Lotina-Hennsen B (2005) Selective Herbicide Activity of 2,5-Di(benzylamine)-p-benzoquinone agains the Monocot Weed Echinochloa crusgalli. An in vivo Analysis of Photosynthesis and Growth. J Agric Food Chem 53: 3415-3420.

26. Hasegawa T, Mochida T, Kondo R, Kagoshima K, Iwasa Y (2000) Mixed-stack organic charge-transfer complexes with intercolumnar networks. Phys Rev B Condens Matter Mater Phys 62: 10059.

27. Siemiarczuk A, Mclntosh AR, Te Fu Ho, Stillman MJ, Roach KJ (1983) Intramolecular photochemical electron transfer. 2. Fluorescence studies of linked porphyrin-quinone compounds. J Am Chem Soc 105: 7224-7230.

28. Trumpower BL (1986) Functions of Quinones in Energy Conserving Systems Academic Press, New York.

29. Stowell MHB, McPhillips TM, Rees DC, Soltis SM, Abresch E (1997) Lightinduced structural changes in photosynthetic reaction center: Implications for mechanism of electron-proton transfer. Science $276: 812$

30. Mukherjee T (2000) Photo and radiation chemistry of quinones, Proc Ind Nat Sci Acad 66: 239.

31. Morton RA (1965) Biochemistry of Quinones, New York, Academic Press 433.

32. Nohl H, Jordan W, Youngman RI (1986) Quinones in biology: functions in electron transfer and oxygen activation. Adv Free Rad Biol Med 2: 211.

33. Bauer AW, Kirby WM, Sherris JC, Turck M (1966) Antibiotic susceptibility testing by a standardized single disk method. Am J Clin Pathol 45: 493-496.

34. Sheldrick GM (2008) A short history of SHELX. Acta Crystallogr A 64: 112-122.

35. Bruno IJ, Cole JC, Edgington PR, Kessler M, Macrae CF, et al. (2002) New software for searching the Cambridge Structural Database and visualizing 
Citation: Gautam BPS, Dani RK, Prasad RL, Srivastava M, Yadav RA, et al. (2015) Synthesis, Characterization, Single Crystal Structural Studies, Antibacterial Activity and DFT Investigations of 2-Chloro-5-Ethoxy-3,6-Bis(Methylamino)-1,4-Benzoquinone. Pharm Anal Acta 6: 418. doi:10.4172/21532435.1000418

crystal structures. Acta Crystallogr B 58: 389-397.

36. Nakamoto K (1986) Infrared and Raman Spectra of Inorganic and Coordination Compounds (6th edn) Wiley Interscience, New York.

37. Lana EJ, Carazza F, Takahashi JA (2006) Antibacterial evaluation of 1,4-benzoquinone derivatives. J Agric Food Chem 54: 2053-2056.

38. Grennberg H, Gogoll A, Backvall JE (1991) Sulfoxide 4 has been demonstrated to accelerate the 1, 4-oxidation of dienes. J Org Chem 56: 5808

39. Martínez MA, Martínez JAB, Ruvalcaba NM, González I, Tovar E (2001) Molecular Structure of Substituted Phenylamine a-OMe- and a-OH-pBenzoquinone Derivatives. Synthesis and Correlation of Spectroscopic, Electrochemical, and Theoretical Parameters. J Org Chem 66: 8349.

40. Illos RA, Shamir D, Shimon LJW, Zilbermann I, Bittner S (2006) N-Dansylcarbazoloquinone; a chemical and electrochemical fluorescent switch. Tetrahedron Lett 47: 5543.

41. Frisch MJ, Trucks GW, Schlegel HB, Scuseria GE, Robb MA (2003) Technical Support Information, Gaussian 03, Revision A.1, Gaussian, Inc., Pittsburgh.

42. Becke AD (1993) Density functional thermochemistry. III. The role of exact exchange. J Chem Phys 98: 5648.

43. Lee C, Yang W, Parr RG (1988) Development of the Colle-Salvetti correlationenergy formula into a functional of the electron density. Phys Rev B Condens Matter 37: 785-789.

44. Dani RK, Bharty MK, Kushawaha SK, Om Prakash, Sharma VK (2014) Syntheses, spectral and structural characterization of trinuclear and mononuclear $\mathrm{Zn}(\mathrm{II})$ complexes of N'-benzoyl hydrazine carboperthioate and 5-phenyl-1,3,4-oxadiazole-2-thione: An approach to DFT calculation, antibacterial and thermal studies. Polyhedron 81: 261.

45. Cash GG, Pettersen RC (1978) The X-ray structure of [Me $3 \mathrm{SiC} 4 \mathrm{SiMe} 3]$ $2 \mathrm{Fe} 2$ (CO) 7: a s-p-type bonding complex between a diacetylene and Iron carbonyl, Acta Cryst B34: 3697.

46. Prasad RL, Kushwaha A, Suchita, Kumar M, Yadav RA (2008) Infrared and ab initio studies of conducting molecules: 2,5-diamino-3,6-dichloro-1,4benzoquinone. Spectrochim Acta A Mol Biomol Spectrosc 69: 304-311.

47. Yadav RA, Shanker R (1987) Raman and infrared spectra and fundamental frequencies of 1,2,4,5-tetrabromobenzene. J Raman Spectrosc 18: 555.

48. Shanker R, Yadav RA, Singh IS, Singh ON (1992) Vibrational spectra, thermodynamic functions and barriers to internal rotation for isomeric trifluoromethylbenzoyl chlorides. J Raman Spectrosc 23: 141.

49. Singh DN, Shanker R, Yadav RA (1996) IR and Raman spectra, force fields, barrier heights and thermodynamic functions for isomeric trifluoromethyl anilines. J Raman Spectrosc 27: 177.
50. Singh DN, Singh JS, Yadav RA (1997) Force Fields for Cand24XXXw $\mathrm{N}$-Substituted Benzenes. II-Planar and Non-Planar Modes of the Three Isomeric Dicyanobenzenes. J Raman Spectrosc 28: 355.

51. Rastogi VK, Jain V, Yadav RA, Singh C, Palafox MA (2000) Fourier transform Raman spectrum and $a b$ initio and density functional computations of the vibrational spectrum, molecular geometry, atomic charges and some molecular properties of the anti-carcinogenic drug 5-fluorouracil. J Raman Spectrosc 31: 595

52. Singh R, Yadav RA (2014) Raman and IR studies and DFT calculations of the vibrational spectra of 2,4-Dithiouracil and its cation and anion. Spectrochim Acta A Mol Biomol Spectrosc 130: 188-197.

53. Yadav RA, Shankar R, Singh IS (1985) Polarized Raman and Infrared-Spectra of 2-Chloro-6-Methylaniline, Indian. J Pure Appl Phys 23: 343.

54. Tanak H (2011) Crystal structure, spectroscopy, and quantum chemical studies of (E)-2-[(2-chlorophenyl)iminomethyl]-4-trifluoromethoxyphenol. J Phys Chem A 115: 13865-13876.

55. Murray JS, Sen K (1996) Molecular Electrostatic Potentials, Concepts and Applications, Elsevier, Amsterdam.

56. Govindarajan M, Karabacak M, Suvitha A, Periandy S (2012) FT-IR, FT-Raman, ab initio, HF and DFT studies, NBO, HOMO-LUMO and electronic structure calculations on 4-chloro-3-nitrotoluene. Spectrochim Acta A 89: 137-148.

57. Arivazhagan M, Meenakshi R (2012) Vibrational spectroscopic studies and DFT calculations of 4-bromo-o-xylene. Spectrochim Acta A Mol Biomol Spectrosc 91: 419-430.

58. Kosar B, Albayrak C (2011) Spectroscopic investigations and quantum chemical computational study of (E)-4-methoxy-2-[(p-tolylimino)methyl]phenol. Spectrochim Acta A 78: 160.

59. Kurtz SK, Perry TT (1968) A Powder Technique for the Evaluation of Nonlinear Optical Materials. J Appl Phys 39: 3798.

60. Fukui K (1982) Role of frontier orbitals in chemical reactions. Science 218 747-754.

61. Khan FA, Choudhury S (2010) Synthesis and electrochemical properties of substituted para-benzoquinone derivatives. Tetrahedron Lett 51: 2541.

62. Dani RK, Bharty MK, Kushawaha SK, Om Prakash, Singh RK (2013) Ni(II), $\mathrm{Cu}(\mathrm{II})$ and $\mathrm{Zn}(\mathrm{II})$ complexes of (Z)-N'(1,3,4-thiadiazol-2-yl) acetimidate: Synthesis, spectral, solid state electrical conductivity, X-ray diffraction and DFT study. Polyhedron, 65: 31.

63. Kushawaha SK, Dani RK, Bharty MK, Chaudhari UK, Sharma VK (2014) Studies on N-picolinoyl-N'-benzothioylhydrazide and its Zn(II) complex: Synthesis structure, antibacterial activity, thermal analysis and DFT calculation, Journal of Molecular Structure 1063: 60. 